\title{
A Novel Transformation Approach of Shared-link Coded Caching Schemes for Multiaccess Networks
}

\author{
Minquan Cheng, Kai Wan, Member, IEEE, Dequan Liang, Mingming Zhang, and Giuseppe Caire, Fellow, IEEE
}

\begin{abstract}
This paper considers the multiaccess coded caching systems formulated by Hachem et al., including a central server containing $N$ files connected to $K$ cache-less users through an error-free shared link, and $K$ cache-nodes, each equipped with a cache memory size of $M$ files. Each user has access to $L$ neighbouring cache-nodes with a cyclic wrap-around topology. The coded caching scheme proposed by Hachem et al. suffers from the case that $L$ does not divide $K$, where the needed number of transmissions (a.k.a. load) is at most four times the load expression for the case where $L$ divides $K$. Our main contribution is to propose a novel transformation approach to smartly extend the schemes satisfying some conditions for the well known shared-link caching systems to the multiaccess caching systems. Then we can get many coded caching schemes with different subpacketizations for multiaccess coded caching system. These resulting schemes have the maximum local caching gain (i.e., the cached contents stored at any $L$ neighbouring cache-nodes are different such that the number of retrieval packets by each user from the connected cache-nodes is maximal) and the same coded caching gain as the original schemes. Applying the transformation approach to the well-known shared-link coded caching scheme proposed by Maddah-Ali and Niesen, we obtain a new multiaccess coded caching scheme that achieves the same load as the scheme of Hachem et al. but for any system parameters. Under the constraint of the cache placement used in this new multiaccess coded caching scheme, our delivery strategy is approximately optimal when $K$ is sufficiently large. Finally, we also show that the transmission load of the proposed scheme can be further reduced by compressing the multicast message.
\end{abstract}

\section{Index Terms}

Coded caching, multiaccess networks, placement delivery array.

\section{INTRODUCTION}

W IRELESS networks are increasingly under stress because of the ever-increasing large number of wireless devices consuming high-quality multimedia content (e.g., on-demand video streaming). The high temporal variability of network traffic results in congestions during the peak traffic times and underutilization of the network during off-peak traffic times. Caching can effectively shift traffic from peak to off-peak times [1], by storing fractions of popular content at users' local memories during the peak traffic times, such that users can be partly served from their local caches, thereby reducing the network traffic.

Coded caching was originally proposed by Maddah-Ali and Niesen (MN) in [2] for the shared-link broadcast network, where a single server with access to a library containing $N$ equal-length files is connected to $K$ users through a shared link. Each of the $K$ users has a cache memory which can store up to $M$ files. A coded caching scheme operates in two phases. In the placement phase, the server populates the users' caches without knowledge of future demands. In the delivery phase, each user demands one file. According to users' demands and caches, the server broadcasts coded messages through the shared link to all users such that each user's demand is satisfied. The goal is minimize the worst-case number of transmissions normalized by the file size (referred to as worst-case load or just load) in the delivery phase among all possible demands. The MN coded caching scheme uses a combinatorial design in the placement phase, such that in the delivery phase each message broadcasted by the server can simultaneously satisfy multiple users' demands. The achieved load of the MN scheme is

$$
R_{\mathrm{MN}}=\frac{K(1-M / N)}{K M / N+1}, \forall M=\frac{N t}{K}: t \in\{0,1, \ldots, K\}
$$

When $N \geq K$, the MN scheme was showed to be optimal under uncoded placement (i.e., each user directly copies some bits of files in its cache) [3], [4] and to be generally order optimal within a factor of 2 [5].

A main drawback of the MN scheme is its high subpacketization. The first coded caching scheme with reduced subpacketization was proposed in [6] with a grouping strategy. The authors in [7] proposed a class of caching schemes based on the concept of placement delivery array (PDA), and showed that the MN scheme is a special PDA, referred to as MN PDA. Based on the concept of PDA, various caching schemes were proposed, e.g., [7]-[14], in order to reduce the subpacketization of the MN scheme. 


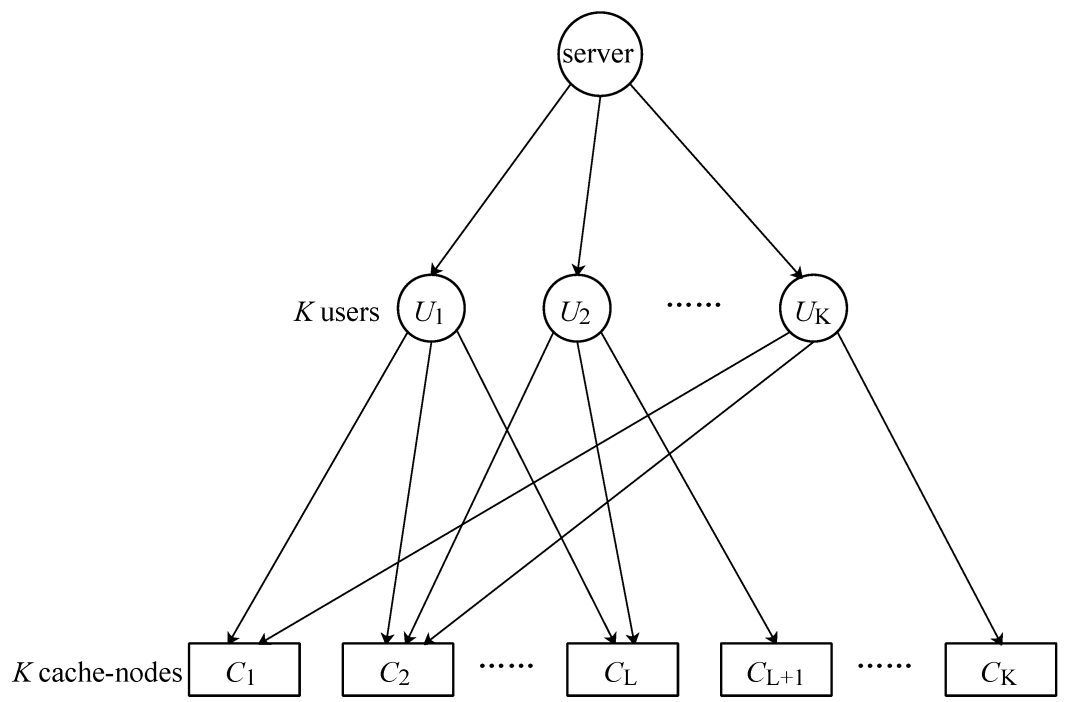

Fig. 1: The $(K, L, M, N)$ multiaccess coded caching system.

\section{A. Multiaccess caching}

Caching at the wireless edge nodes is a promising way to boost the spatial and spectral efficiency, for the sake of reducing communication cost of wireless systems [15], [16]. Edge caches enable storing Internet-based content, including web objects, videos and software updates. Compared to the end-user-caches, which are heavily limited by the storage size of each user's device (e.g., a library size is usually much larger than the storage size of a mobile device) and only useful to one user, the caches at edge devices such as local helpers, mobile edge computing (MEC) servers and hotspots, can be made much larger and can be accessed my multiple users. In this paper, we consider an ideal coded edge caching model with a line multiaccess topology, referred to as multiaccess caching model (illustrated in Fig. 1], which was originally introduced in [17]. Different from the shared-link caching system, in the multiaccess caching system, there are $K$ cache-nodes each of storage $M$ files, and $K$ cache-less users each of which can access $L$ cache-nodes in a cyclic wrap-around fashion, while also receiving the broadcast transmission directly from the server. As assumed in [17], the cache-nodes in the considered model are accessed at no load cost; that is, we count only the broadcasted load of the server. This can be justified by the fact that the access to the cache-nodes can be offloaded on a different network (e.g., a wifi offloading for hotspots).

A multiaccess coded caching scheme was proposed in [17], which deals with the following two cases separately.

- When $L$ divides $K$ (i.e., $L \mid K$ ), the authors in [17] proposed a scheme that divides the $K$ users into $L$ groups, where the $\mathrm{MN}$ scheme is then used in each user group. This results in a total load equal to

$$
R_{\mathrm{HKD}}=\frac{K(1-L M / N)}{K M / N+1}, \forall M=\frac{N t}{K}: t \in\left\{0,1, \ldots,\left\lfloor\frac{K}{L}\right\rfloor\right\} .
$$

In this case, the multiplicative gap between the resulting scheme and the MN scheme (with the memory ratio $\frac{L M}{N}$ ) is at most $L 1$

- When $L$ does not divide $K$, the authors showed that a load which is three times larger than the one in (2) can be achieved. The authors in [18] proposed a new scheme based on erasure-correcting code which has lower load than the scheme in [17] when $K<\frac{K L M}{N}+L$. In addition, when $L \geq \frac{K}{2}$, by extending the converse bound in [3], a converse bound under uncoded cache placement was also proposed in [18]. Under the constraint of $N \geq K, L \geq \frac{K}{2}$, and uncoded cache placement, the scheme in [18] was shown to be order optimal within a factor of 2. For some special parameters of the multiaccess coded caching problem, some improved schemes with reduced load compared to the scheme in [18] were proposed in [19]-[22]. The authors in [23] extended the linear topology multiaccess coded caching scheme [17] to two-dimensional cellular networks, where the mobile users move on a two-dimensional grid and can access the nearest cache-nodes inside the grid.

M. Cheng, D. Liang, and M. Zhang are with Guangxi Key Lab of Multi-source Information Mining \& Security, Guangxi Normal University, Guilin 541004, China (e-mail: chengqinshi@hotmail.com,dequan.liang@hotmail.com,ztw_07@foxmail.com).

K. Wan and G. Caire are with the Electrical Engineering and Computer Science Department, Technische Universität Berlin, 10587 Berlin, Germany (e-mail: kai.wan@tu-berlin.de, caire@tu-berlin.de). The work of K. Wan and G. Caire was partially funded by the European Research Council under the ERC Advanced Grant N. 789190, CARENET.

${ }^{1}$ Notice that if each user has an individual cache of size $L M$, then the MN scheme would achieve the load $\frac{K(1-L M / N)}{K L M / N+1}$. Instead, if each user has an individual cache of size $M$, then the MN scheme would achieve the load in (1). Therefore, the multiaccess case model here where each user accesses to $L$ neighbouring cache-nodes, yields an intermediate performance in between the two extremes. 


\section{B. Contribution and paper organization}

This paper focuses on the multiaccess caching system in [17]. We show that any PDA satisfying two conditions defined in Section IV] (e.g., the MN PDA and the PDAs in [7], [10], [12], [13]) can be used to construct a multiaccess coded caching scheme by a novel three-step transformation. The resulting scheme has the maximum local caching gain (i.e., the cached contents stored at any $L$ neighbouring cache-nodes are different such that each user can totally retrieve $L M$ files from the connected cache-nodes) and the same coded caching gain as the shared-link coded caching scheme realized by the original PDA. Interestingly, the load of the resulting multiaccess coded caching scheme obtained by using the MN PDA achieves the same load as in (2) for any system parameters, i.e., without constraint that $L$ divides $K$ in [17]. Under the constraint of the cache placement used in this multiaccess coded caching scheme, we show that the delivery scheme is approximately optimal when $K$ is sufficiently large. Furthermore we can reduce the load of our resulting schemes in delivery phase by further compressing the multicast messages.

The rest of this paper is organized as follows. The multiaccess coded caching model and some preliminary results are introduced in Section III] The main results of this paper are listed in Section III] Section IV] provides the detailed construction of the proposed caching scheme. An further improved transformation approach is described in Section $\overline{\mathrm{V}}$. Finally, we conclude the paper in Section VI, and some proofs are provided in the Appendices.

\section{Notations}

- Bold capital letter, bold lower case letter and curlicue font will be used to denote array, vector and set respectively. We assume that all the sets are increasing ordered and $|\cdot|$ is used to represent the cardinality of a set or the length of a vector;

- For any positive integers $a, b, t$ with $a<b$ and $t \leq b$, and any nonnegative set $\mathcal{V}$,

- let $[a: b]=\{a, a+1, \ldots, b\}$, especially $[1: b]$ be shorten by $[b]$, and $\left(\begin{array}{c}{[b]} \\ t\end{array}\right)=\{\mathcal{V}|\mathcal{V} \subseteq[b],| \mathcal{V} \mid=t\}$, i.e., $\left(\begin{array}{c}{[b]} \\ t\end{array}\right)$ is the collection of all $t$-sized subsets of $[b]$;

- Mod $(b, a)$ represents the modulo operation on positive integer $b$ with positive integer divisor $a$. In this paper we let $\operatorname{Mod}(b, a) \in\{1, \ldots, a\}$ (i.e., we let $\operatorname{Mod}(b, a)=a$ if $a$ divides $b) . \mathcal{V}[h]$ represent the $h^{\text {th }}$ smallest element of $\mathcal{V}$, where $h \in[|\mathcal{V}|]$;

$-\operatorname{Mod}(\mathcal{V}, a)=\{\operatorname{Mod}(\mathcal{V}[h], a): h \in[|\mathcal{V}|]\}$

$-\mathcal{V}+a=\{\mathcal{V}[h]+a: h \in[|\mathcal{V}|]\}$.

- the array $[a ; b]$ is written in a Matlab form, representing $\left[\begin{array}{l}a \\ b\end{array}\right]$.

\section{System Model AND Related Works}

In this section, we first introduce the shared-link caching system in [2], and the $\mathrm{MN}$ caching scheme from the viewpoint of placement delivery array (PDA) [7]. Then the multiaccess caching model and previously known results are introduced.

\section{A. The original caching model}

In the shared-link coded caching system [2], a server containing $N$ files with equal length in $\mathcal{W}=\left\{W_{1}, W_{2}, \ldots, W_{N}\right\}$ connects through an error-free shared link to $K$ users in $\left\{U_{1}, U_{2}, \ldots, U_{K}\right\}$ with $K \leq N$, and every user has a cache which can store up to $M$ files for $0 \leq M \leq N$. An $F$-division $(K, M, N)$ coded caching scheme contains two phases.

- Placement phase: Each file is divided into $F$ packets with equal size ${ }^{2}$ and then each user $U_{k}$ where $k \in[K]$ caches some packets of each file, which is limited by its cache size $M$. Let $\mathcal{Z}_{U_{k}}$ denote the cache contents at user $U_{k}$, which is assumed to be known to the server. Notice that the placement phase is done without knowledge of later requests.

- Delivery phase: Each user randomly requests one file from the server. The requested file by user $U_{k}$ is represented by $W_{d_{U_{k}}}$, and the request vector by all users is denoted by $\mathbf{d}=\left(d_{U_{1}}, d_{U_{2}}, \ldots, d_{U_{K}}\right)$. According to the cached contents and requests of all the users, the server transmits a broadcast message including $S_{\mathbf{d}}$ packets to all users, such that each user's request can be satisfied.

In such system, the number of worst-case transmitted files (a.k.a. load) for all possible requests is expected to be as small as possible, which is defined as

$$
R=\max _{\mathbf{d} \in[N]^{K}} \frac{S_{\mathbf{d}}}{F} .
$$

For the shared-link coded caching problem, the authors in [7] proposed a class of solutions based on the concept of placement delivery array (PDA) which is defined as follows.

Definition 1. ( [7]) For positive integers $K, F, Z$ and $S$, an $F \times K$ array $\mathbf{P}=\left(p_{j, k}\right)_{j \in[F], k \in[K]}$, composed of a specific symbol "*" and $S$ positive integers from $[S]$, is called a $(K, F, Z, S)$ PDA if it satisfies the following conditions:

\footnotetext{
${ }^{2}$ In this paper, we only consider the uncoded cache placement.
} 
C1. The symbol "*" appears $Z$ times in each column;

C2. Each integer occurs at least once in the array;

C3. For any two distinct entries $p_{j_{1}, k_{1}}$ and $p_{j_{2}, k_{2}}, p_{j_{1}, k_{1}}=p_{j_{2}, k_{2}}=s$ is an integer only if

a. $j_{1} \neq j_{2}, k_{1} \neq k_{2}$, i.e., they lie in distinct rows and distinct columns; and

b. $p_{j_{1}, k_{2}}=p_{j_{2}, k_{1}}=*$, i.e., the corresponding $2 \times 2$ subarray formed by rows $j_{1}, j_{2}$ and columns $k_{1}, k_{2}$ must be of the following form

$$
\left(\begin{array}{ll}
s & * \\
* & s
\end{array}\right) \text { or }\left(\begin{array}{ll}
* & s \\
s & *
\end{array}\right) \text {. }
$$

In a $(K, F, Z, S)$ PDA $\mathbf{P}$, each column represents one user's cached contents, i.e., if $p_{j, k}=*$, then user $U_{k}$ has cached the $j^{t h}$ packet of all the files. If $p_{j, k}=s$ is an integer, it means that the $j^{\text {th }}$ packet of all the files is not stored by user $U_{k}$. Then the XOR of the requested packets indicated by $s$ is broadcasted by the server at time slot $s$. The property C2 of Definition 1 implies that the number of signals transmitted by the server is exactly $S$. So the load is $R=\frac{S}{F}$. Finally the property C3 of Definition 1 guarantees that each user can get the requested packet, since it has cached all the other packets in the signal except its requested one. Hence, the following lemma was proved by Yan et al. in [7].

Lemma 1. ( [7] ) Using Algorithm 1, an $F$-division caching scheme for the $(K, M, N)$ caching system can be realized by a $(K, F, Z, S)$ PDA with $\frac{M}{N}=\frac{Z}{F}$. Each user can decode his requested file correctly for any request $\mathbf{d}$ at the rate $R=\frac{S}{F}$.

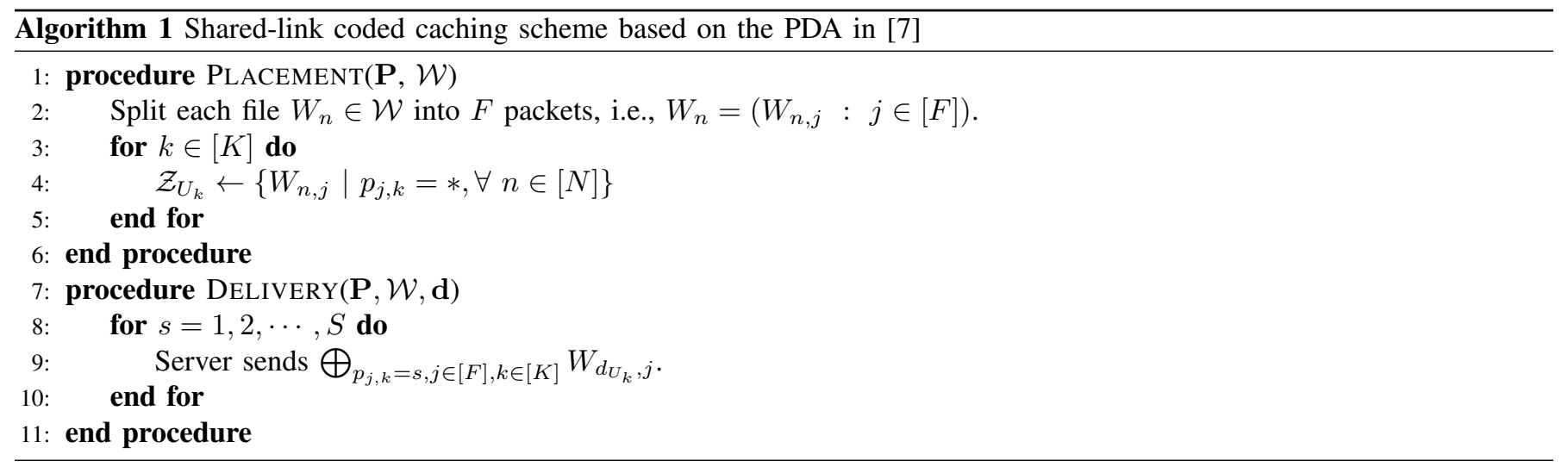

Let us briefly introduce the MN coded caching scheme in [2] from the viewpoint of MN PDA, where the resulting PDA is referred to as MN PDA. For any integer $t \in[K]$, we let $F=\left(\begin{array}{c}K \\ t\end{array}\right)$. We arrange all the subsets with size $t+1$ of $[K]$ in the lexicographic order and define $\phi(\mathcal{S})$ to be its order for any subset $\mathcal{S}$ of size $t+1$. Clearly, $\phi$ is a bijection from $\left(\begin{array}{l}{[K]} \\ t+1\end{array}\right)$ to $\left[\left(\begin{array}{c}K \\ t+1\end{array}\right)\right]$. Then, an MN PDA is defined as a $\left(\begin{array}{c}K \\ t\end{array}\right) \times K$ array $\mathbf{P}=\left(p_{\mathcal{T}, k}\right)_{\mathcal{T} \in\left(\begin{array}{c}{[K]} \\ t\end{array}\right), k \in[K]}$ by

$$
p_{\mathcal{T}, k}=\left\{\begin{array}{cc}
\phi(\mathcal{T} \cup\{k\}), & \text { if } k \notin \mathcal{T} \\
*, & \text { otherwise }
\end{array}\right.
$$

where the rows are labelled by all the subsets $\mathcal{T} \in\left(\begin{array}{c}{[K]} \\ t\end{array}\right)$. Thus, by the definition of PDA, the achieved load of the MN PDA is as follows.

Lemma 2. (MN PDA [2]) For any positive integers $K$ and $t$ with $t<K$, there exists a $\left(K,\left(\begin{array}{c}K \\ t\end{array}\right),\left(\begin{array}{c}K-1 \\ t-1\end{array}\right),\left(\begin{array}{c}K \\ t+1\end{array}\right)\right)$ PDA, which gives a $\left(\begin{array}{c}K \\ t\end{array}\right)$-division $(K, M, N)$ coded caching scheme for the shared-link caching system with the memory ratio $\frac{M}{N}=\frac{t}{K}$ and load $R=\frac{K-t}{t+1}$.

Example 1. We then illustrate the MN PDA by the following example where $N=K=4$ and $M=2$. By the construction of the MN PDA, we have the following $(4,6,3,4)$ PDA.

$$
\mathbf{P}=\left(\begin{array}{cccc}
* & * & 1 & 2 \\
* & 1 & * & 3 \\
* & 2 & 3 & * \\
1 & * & * & 4 \\
2 & * & 4 & * \\
3 & 4 & * & *
\end{array}\right)
$$

Using Algorithm 1, the detailed caching scheme is as follows. 
- Placement Phase: From Line 2 we have $W_{n}=\left(W_{n, 1}, W_{n, 2}, W_{n, 3}, W_{n, 4}, W_{n, 5}, W_{n, 6}\right)$ where $n \in[4]$. Then by Lines 3-5, the users' caches are

$$
\begin{array}{lll}
\mathcal{Z}_{U_{1}}=\left\{W_{n, 1}, W_{n, 2}, W_{n, 3}: n \in[4]\right\} ; & \mathcal{Z}_{U_{2}}=\left\{W_{n, 1}, W_{n, 4}, W_{n, 5}: n \in[4]\right\} ; \\
\mathcal{Z}_{U_{3}}=\left\{W_{n, 2}, W_{n, 4}, W_{n, 6}: n \in[4]\right\} ; & \mathcal{Z}_{U 4}=\left\{W_{n, 3}, W_{n, 5}, W_{n, 6}: n \in[4]\right\}
\end{array}
$$

- Delivery Phase: Assume that the request vector is $\mathbf{d}=(1,2,3,4)$. By the transmitting process by Lines $8-10$, the server transmits the following multicast messages with total load $R=\frac{4}{6}=\frac{2}{3}$.

\begin{tabular}{|c|l|}
\hline Time Slot & Transmitted Signnal \\
\hline 1 & $W_{1,4} \oplus W_{2,2} \oplus W_{3,1}$ \\
\hline 2 & $W_{1,5} \oplus W_{2,3} \oplus W_{4,1}$ \\
\hline 3 & $W_{1,6} \oplus W_{3,3} \oplus W_{4,2}$ \\
\hline 4 & $W_{2,6} \oplus W_{3,5} \oplus W_{4,4}$ \\
\hline
\end{tabular}

TABLE I: Delivery steps in Example 1

\section{B. Multiaccess coded caching model}

We then introduce the $(K, L, M, N)$ multiaccess coded caching problem in [17] (as illustrated in Fig. 1), containing a server with a set of $N$ equal-length files (denoted by $\mathcal{W}=\left\{W_{1}, \ldots, W_{N}\right\}$ ), $K$ cache-nodes (denoted by $C_{1}, \ldots, C_{K}$ ), and $K \leq N$ users (denoted by $U_{1}, \ldots, U_{K}$ ). Each cache-node has a memory size of $M$ files where $0 \leq M \leq \frac{N}{L}$. Each user is connected to $L$ neighbouring cache-nodes in a cyclic wrap-around fashion, where user $U_{k}$ can access the cache-nodes $C_{k}, C_{\operatorname{Mod}(k+1, K)}, \ldots, C_{\operatorname{Mod}(k+L-1, K)}$ for each $k \in[K]$. Each user is also connected via an error-free shared link to the server. In this paper, we assume that the communication bottleneck is on the shared link from the server to the users; thus we assume that each user can retrieve the cached contents from its connected cache-nodes without any cost.

An $F$-division $(K, L, M, N)$ multiaccess coded caching scheme runs in two phases,

- Placement phase: each file is divided into $F$ packets of equal size, and then each cache-node $C_{k}$ where $k \in[K]$, directly caches some packets of each file, which is limited by its cache size $M$. Let $\mathcal{Z}_{C_{k}}$ denote the cache contents at cache-node $C_{k}$. The placement phase is also done without knowledge of later requests. Each user $U_{k}$ where $k \in[K]$ can retrieve the contents cached at the $L$ neighbouring cache-nodes in a cyclic wrap-around fashion. Let $\mathcal{Z}_{U_{k}}$ denote the retrievable cache contents by user $U_{k}$.

- Delivery phase: each user randomly requests one file. According to the request vector $\mathbf{d}=\left(d_{U_{1}}, d_{U_{2}}, \ldots, d_{U_{K}}\right)$ and the cached contents in the cache-nodes, the server transmits $S_{\mathbf{d}}$ multicast messages to all users, such that each user's request can be satisfied.

We aim to design a multiaccess coded caching scheme with minimum worst-case load as defined in (3).

Note that any uncoded cache placement (as described above), we can divide each file $W_{n}, n \in[N]$ into subfiles, $W_{n}=$ $\left\{W_{n, \mathcal{T}}: \mathcal{T} \subseteq[K]\right\}$. Subfile $W_{n, \mathcal{T}}$ represents the set of packets of $W_{n}$ cached by cache-nodes $C_{k}$ where $k \in \mathcal{T}$.

The first multiaccess coded caching scheme was proposed in [17], where the following result was proved.

Lemma 3 ( [17]). For the $(K, L, M, N)$ multiaccess coded caching problem,

- when $L \mid K$, the lower convex envelope of the memory-load tradeoff points

$$
\left(M, R_{\mathrm{HKD}}\right)=\left(\frac{N t}{K}, \frac{K-t L}{t+1}\right), \forall t \in\left\{0,1, \ldots,\left\lfloor\frac{K}{L}\right\rfloor\right\}
$$

and $\left(M, R_{\mathrm{HKD}}\right)=\left(\frac{N}{L}, 0\right)$, is achievable.

- When $L \nmid K$, the lower convex envelope of the memory-load tradeoff points

$$
\left(M, R_{\mathrm{HKD}}\right)=\left(\frac{N t}{K}, \frac{K-t}{t+1}\right), \forall t \in\left\{0,1, \ldots,\left\lfloor\frac{K}{2 L}\right\rfloor\right\}
$$

and $\left(M, R_{\mathrm{HKD}}\right)=\left(\frac{N}{L}, 0\right)$, is achievable.

When $L \mid K$, the authors in [17] showed that the multiplicative gap between the $R_{\mathrm{HKD}}$ and the MN scheme with the memory ratio $\frac{L M}{N}$ is at most $L$. Based on Minimum Distance Separable (MDS) codes, the authors in [18] proposed the following improved scheme. 
Lemma $4([18])$. For the $(K, L, M, N)$ multiaccess coded caching problem, the lower convex envelope of the memory-load tradeoff points

$$
\left(M, R_{\mathrm{RK}}\right)=\left(\frac{N t}{K}, \frac{(K-t L)^{2}}{K}\right), \forall t \in\left\{0,1, \ldots,\left\lfloor\frac{K}{L}\right\rfloor\right\}
$$

is achievable.

The load in (8) was shown in [18] to be optimal under uncoded cache placement when $L=K-1 ; L=K-2 ; L=K-3$ for $K$ is even. The authors in [19] proposed a scheme with the subpacketization $F=K(K-1)$ achieving the following load.

Lemma 5 ( [19]). For the $(K, L, M, N)$ multiaccess coded caching problem, the lower convex envelope of the memory-load tradeoff points $\left(\frac{N t}{K}, R_{\mathrm{SR}}\right)$ for all $t \in\left\{0,1, \ldots,\left\lfloor\frac{K}{L}\right\rfloor\right\}$ and $\left(\frac{N}{L}, 0\right)$, is achievable, where

- $R_{\mathrm{SR}}=\frac{1}{K}$ if $K-1=t L$;

- $R_{\mathrm{SR}}=\sum_{h=\frac{K-t L+2}{2}}^{K-t L} \frac{2}{1+\left\lceil\frac{t L}{h}\right\rceil}$ if $K-t L$ is even;

- $R_{\mathrm{SR}}=\frac{1}{\left\lceil\frac{2 t L}{K-t L+1}\right\rceil+1}+\sum_{h=\frac{K-t L+3}{2}}^{2} \frac{2}{1+\left\lceil\frac{t L}{h}\right\rceil}$ if $K-t L>1$ is odd.

When $M=\frac{2 N}{K}$ (i.e., $t=\frac{K M}{N}=2$ ), the authors in [20] proposed a caching scheme with load $\frac{K-t L}{g}$ where $g>t+1=3$, which is strictly lower than the load in $[6]$. When $\bar{M}=\frac{N}{K}$, the authors in $[21]$ proposed a caching scheme with linear subpacketization, but achieving a higher load than the above schemes when $L<\frac{\Pi}{2}$.

\section{MAIN RESULTS}

\section{A. Main results and performance analysis}

In this section, a novel multiaccess coded caching scheme can be obtained by a non-trivial transformation from an appropriate PDA satisfying some properties. By applying the novel transformation to the MN PDA, we obtain the following theorem, whose detailed proof could be found in Section IV

Theorem 1. For the $(K, L, M, N)$ multiaccess coded caching problem, the lower convex envelope of the following memoryload tradeoff corner points are achievable,

$$
\left(M, R_{1}\right)=\left(\frac{N t}{K}, \frac{K-t L}{t+1}\right), \forall t \in\left[0:\left\lfloor\frac{K}{L}\right\rfloor\right],
$$

and $\left(M, R_{1}\right)=\left(\frac{N}{L}, 0\right)$. The subpacketization is $F=K\left(\begin{array}{c}K-t(L-1) \\ t\end{array}\right)$ when $M=\frac{N t}{K}$, for $t \in\left[0:\left\lfloor\frac{K}{L}\right\rfloor\right]$.

Note that the scheme in Theorem 1 achieves the same load as the scheme in [17] but for any parameters $K$ and $L$. As the existing schemes in Lemmas 3, 5 , the non-trivial corner points of the proposed scheme are at the memory sizes $M=\frac{N t}{K}$ where $t \in\left\{1, \ldots,\left\lfloor\frac{K}{L}\right\rfloor\right\}$.

We then compare the achieved load by the proposed scheme in Theorem 1 with the existing schemes in Lemmas 3

- Comparison to Lemma 3 .

1) When $L$ divides $K$, the proposed scheme in Theorem 1 achieves the same load as Lemma 3 , i.e., $R_{1}=R_{\mathrm{HKD}}$.

2) When $L$ does not divide $K$, we have $\frac{R_{\mathrm{HKD}}}{R_{1}}=\frac{K-t}{K-L t}$ for $M=\frac{N t}{K}$ where $t \in\left\{0,1, \ldots,\left\lfloor\frac{K}{2 L}\right\rfloor\right\}$. In addition, for $\frac{N}{K}\left\lfloor\frac{K}{2 L}\right\rfloor \leq M<\frac{N}{L}$, we have

$$
\frac{R_{\mathrm{HKD}}}{R_{1}}>\frac{K-\left\lfloor\frac{K}{2 L}\right\rfloor}{K-L\left\lfloor\frac{K}{2 L}\right\rfloor}
$$

which will be proved in Appendix B-A

- Comparison to Lemma 4. For the non-trivial corner points with $t=\frac{K M}{N} \in\left\{1, \ldots,\left\lfloor\frac{K}{L}\right\rfloor\right\}$, it will be proved in Appendix B-B that

$$
R_{1}<R_{\mathrm{RK}} \text {, if } K>(t+1) L \text {. }
$$

- Comparison to Lemma 5 Due to the high complexity of the closed-form of the load in Lemma 5, we cannot provide the exact comparison between the proposed scheme and the scheme in Lemma 5. Instead, in Appendix B-C we will show that for the non-trivial corner points with $t=\frac{K M}{N} \in\left\{1, \ldots,\left\lfloor\frac{K}{L}\right\rfloor\right\}$, we have

$$
R_{1}<R_{\mathrm{SR}} \text {, if } K \gg L \text {. }
$$


Note that the designed placement of the proposed scheme in Theorem 1 is identical to the placements in [17], [18] in the sense of subfile division. More precisely, for $M=\frac{N t}{K}$ where $t \in\left[\left\lfloor\frac{K}{L}\right\rfloor\right]$, each file $W_{n}, n \in[N]$ is divided into $\left(\begin{array}{c}K-t L+t-1 \\ t-1\end{array}\right) \frac{K}{t}$ non-overlapping and equal-length subfiles, where

$$
W_{n}=\left\{W_{n, \mathcal{T}}: \mathcal{T} \subseteq[K],|\mathcal{T}|=t, \operatorname{Mod}\left(j_{1}-j_{2}, K\right) \geq L, j_{1}, j_{2} \in \mathcal{T} \text { and } j_{1} \neq j_{2}\right\}
$$

in words, each subfile is cached by $t$ cache-nodes and any two cache-nodes within distance $L-1$ do not cache any common packets. The optimal load under the cache placement with subfile division in (13) is denoted by $R_{1}^{\star}$. Under this placement, we propose a converse bound on the load, and prove that when $K \gg t L$ the proposed delivery scheme in Theorem 1 is approximately optimal, where the detailed proof could be found in Appendix A.

Theorem 2. For the $(K, L, M, N)$ multiaccess coded caching problem with $M=\frac{N t}{K}$ where $t \in\left[\left\lfloor\frac{K}{L}\right\rfloor\right]$, it holds that

$$
\begin{aligned}
& R_{1}^{\star} \geq \frac{\left(\begin{array}{c}
K-L-X \\
t-1
\end{array}\right)+(K-L-1)\left(\begin{array}{c}
K-L-X+1 \\
t
\end{array}\right)-\left(\begin{array}{c}
K-L-X \\
t+1
\end{array}\right)}{K\left(\begin{array}{c}
K-X \\
t-1
\end{array}\right)}, \\
& \text { where } X:=t L-t+1 .
\end{aligned}
$$

When $K \gg t L$, we have

$$
\frac{R_{1}}{R_{1}^{\star}} \approx 1 .
$$

It will be explained in Section III-B and Section IV the main novelty of the proposed scheme in Theorem 1 is a smart transformation of the MN PDA for the multiaccess caching problem. In Section IV-C we will point out any PDA satisfying the conditions C4 and C5 in Proposition 1 can be used to generate the scheme for the multiaccess caching problem, e.g., the PDAs in [7], [10], [12], [13]. In one word, based on the original PDA, we design a multiacess coded caching scheme with maximal local caching gain (i.e., any $L$ neighbouring cache-nodes do not cache any same packet such that each user can retrieve $L M F$ packets from its connected cache-nodes) and the same coded caching gain as the original PDA. As a result, our proposed transformation approach has advantages both in terms of load and in terms of subpacketization. For instance, to apply the transformation approach into the PDA in [7], we obtain the following multiaccess coded caching scheme with a lower subpacketization, whose proof is in Appendix D

Theorem 3. For any positive integers $m, q \geq 2$, there exists a $(K=m(q+L-1), L, M, N)$ multiaccess coded caching scheme with the memory ratio $\frac{M}{N}=\frac{1}{q+L-1}$, subpacketization $F=m q^{m-1}(q+L-1)$ and transmission load $R_{2}=q-1$.

We conclude this subsection with some numerical evaluations to compare the proposed schemes in Theorems 1 and 3 , the derived converse in Theorem 2 and the existing schemes in Lemmas 3. 5 . In Fig. 2 and Fig. 3, we consider the multiaccess caching problem with $K=N=20, L=3$, When $L=3$, it can be seen that the proposed scheme performs the best when $0 \leq \frac{M}{N} \leq \frac{K / L-1}{K}=\frac{20 / 3-1}{20}=\frac{1}{3}-\frac{1}{20} \approx 0.28$ by the condition $K>(t+1) L$ in $(11)$. When $L=4$, it can be seen that the proposed scheme performs the best when $0 \leq \frac{M}{N} \leq 0.217$.

\section{B. Sketch of the proposed scheme in Theorem 1}

Let us consider the $(K, L, M, N)=(8,3,2,8)$ multiaccess caching problem where user $U_{k}$ can access the cache-nodes $C_{k}, C_{\operatorname{Mod}(k+1,8)}$ and $C_{\operatorname{Mod}(k+2,8)}$ for each $k \in[8]$. We aim to propose a multiaccess coded caching scheme based on the $\left(K^{\prime}, F^{\prime}, Z, S\right)=(4,6,3,4)$ MN PDA, i.e., $\mathbf{P}$ in (5). It will be clear in Section IV that we choose the MN PDA where $K^{\prime}=K-\frac{K M}{N}(L-1)$ and $K^{\prime} \frac{Z}{F^{\prime}}=K \frac{M}{N}$. Generally speaking, the main ingredients of the proposed scheme contain two key points:

- According to the multiaccess caching model, in order to fully use the cache-nodes, we design a cache placement such that any two cache-nodes connected to some common user(s) should not cache the same packets. Since each user can access $L$ cache-nodes, the local caching gain of the proposed scheme is

$$
g_{\text {local }}=1-\frac{L M}{N}=\frac{1}{4} \text {. }
$$

- The structure of the proposed placement and delivery phases is based on the MN PDA, such that the coded caching gain is the same as the $(4,6,3,4) \mathrm{MN}$ PDA, which is equal to

$$
g_{\text {coded }}=\frac{K^{\prime} Z}{F^{\prime}}+1=\frac{K M}{N}+1=3
$$

Thus the load of the proposed scheme is

$$
K \frac{g_{\text {local }}}{g_{\text {coded }}}=\frac{2}{3}
$$




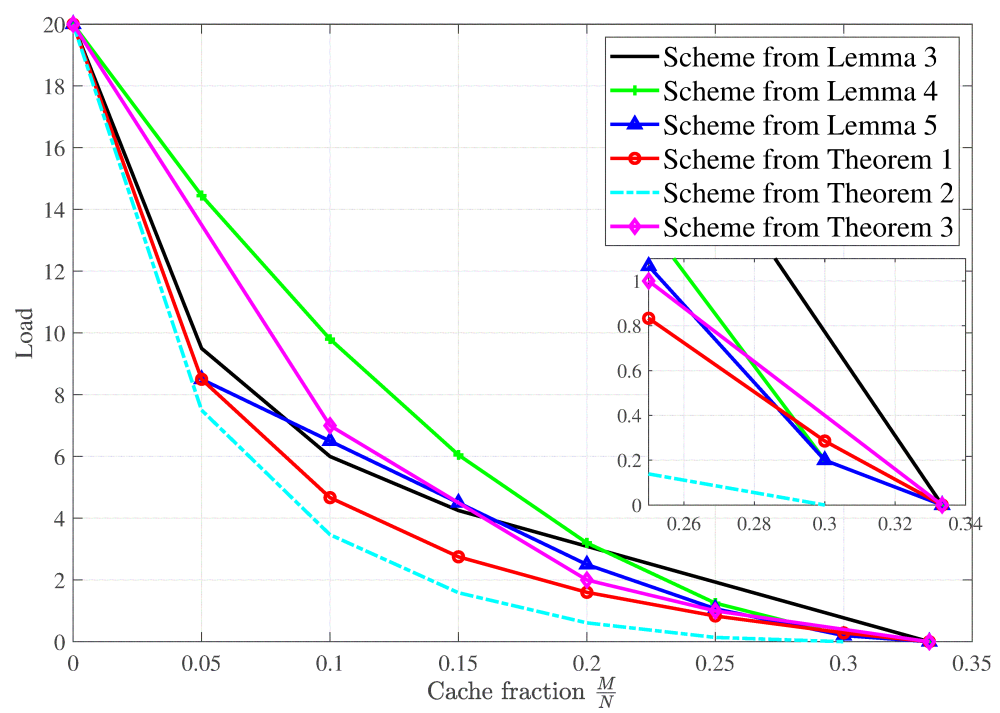

Fig. 2: Comparison of the caching schemes for the multiaccess coded caching problem with $N=K=20$ and $L=3$.

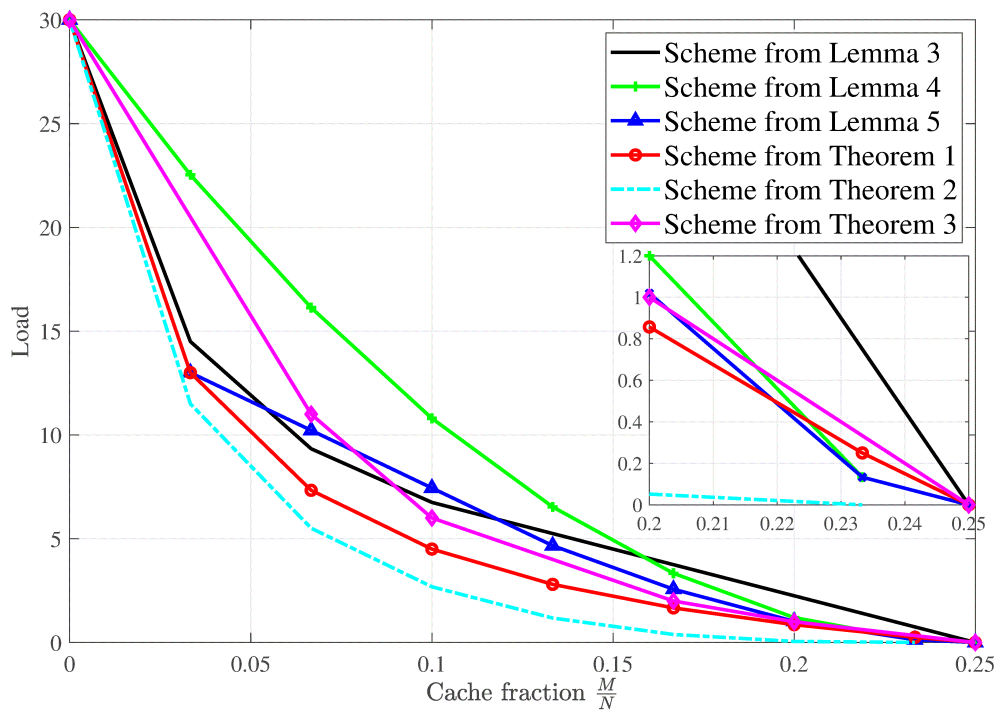

Fig. 3: Comparison of the caching schemes for the multiaccess coded caching problem with $N=K=30$ and $L=4$.

which coincides with (9). Then we introduce the more details on the construction. We divide each file into $K=8$ parts with equal length, $W_{n}=\left(W_{n}^{(g)}: g \in[8]\right)$, and divide the caching procedure into 8 rounds, where in the $g^{\text {th }}$ round we only consider the $g^{\text {th }}$ part of each file. We should point out that in order to ensure all the cache-nodes caching the same amount of contents, we have to divide the caching procedure into $K$ rounds. Furthermore, it will be clarified later that the caching procedures for different rounds are totally symmetric, and thus we only need to design the caching scheme for the first round (i.e., for the first subfiles of the $N$ files).

Let us consider the first round to illustrate the main idea. We further divide the first part of each file (i.e., $W_{n}^{(1)}$ for each $n \in[8]$ ) into $F^{\prime}=6$ non-overlapping and equal-length packets. We then construct three arrays $\mathbf{C}^{(1)}, \mathbf{U}^{(1)}$, and $\mathbf{Q}^{(1)}$ via $\mathbf{P}$, defined as follows.

Definition 2. For each $g \in[K]$,

- An $F^{\prime} \times K$ node-placement array $\mathbf{C}^{(g)}$ consists of star and null, where $F^{\prime}$ and $K$ represent the subpacketization of each part and the number of cache-nodes, respectively. The entry located at the position $(j, k)$ in $\mathbf{C}^{(g)}$ is star if and only if the $k^{\text {th }}$ cache-node caches the $j^{\text {th }}$ packet of each $W_{n}^{(g)}$ where $n \in[N]$.

- An $F^{\prime} \times K$ user-retrieve array $\mathbf{U}^{(g)}$ consists of star and null, where $F^{\prime}$ and $K$ represent the subpacketization of each part and the number of users, respectively. The entry at the position $(j, k)$ in $\mathbf{U}^{(g)}$ is star if and only if the $k^{\text {th }}$ user can 


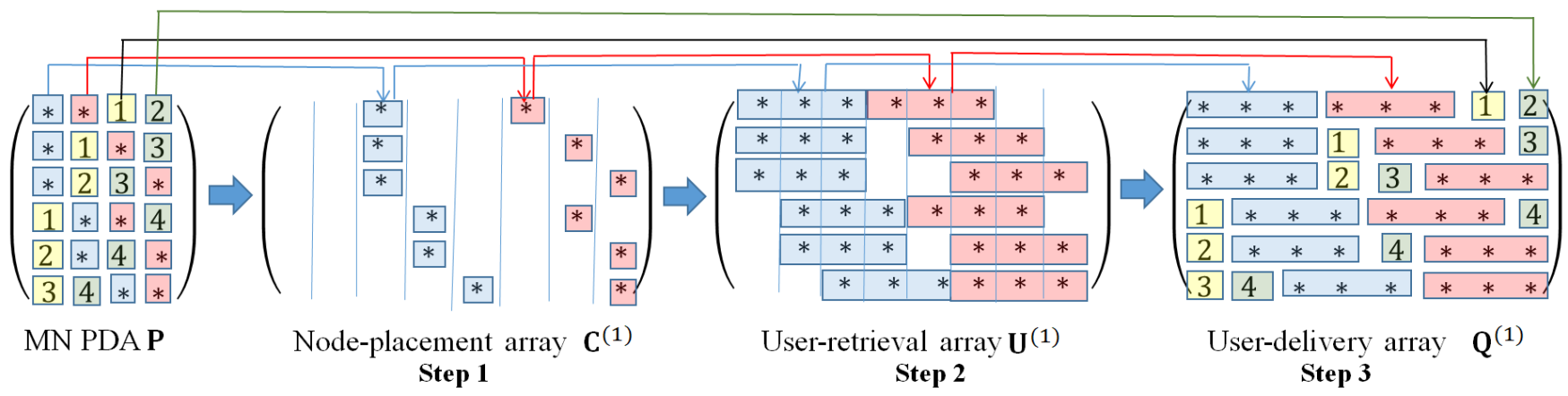

Fig. 4: The flow diagram of constructing $\mathbf{C}^{(1)}, \mathbf{U}^{(1)}$ and $\mathbf{Q}^{(1)}$ via $(4,6,3,4)$ MN PDA

retrieve the $j^{\text {th }}$ packet of each $W_{n}^{(g)}$ where $n \in[N]$.

- An $F^{\prime} \times K$ user-delivery array $\mathbf{Q}^{(g)}$ consists of $\{*\} \cup[S]$, where $F^{\prime}, K$ and the stars in $\mathbf{Q}^{(g)}$ have the same meaning as $F^{\prime}, K$ of $\mathbf{U}^{(g)}$ and the stars in $\mathbf{U}^{(g)}$, respectively. Each integer represents a multicast message, and $S$ represents the total number of multicast messages transmitted in the $g^{\text {th }}$ round of the delivery phase.

The constructing flow diagram from $\mathbf{P}$ to $\mathbf{Q}^{(1)}$ (listed in Fig. 4 contains the following three steps:

- Step 1. In the first step, we construct the node-placement array $\mathbf{C}^{(1)}$ from $\mathbf{P}$. More precisely, let us focus on each row $j \in[6]$ of $\mathbf{P}$. It can be seen that row $j$ contains two stars, which are assumed to be located at columns $k_{1}$ and $k_{2}$, respectively. We let row $j$ of $\mathbf{C}^{(1)}$ also contain two stars, where the first star is located at column $k_{1}+(L-1)=k_{1}+2$ and the second star is located at column $k_{2}+2(L-1)=k_{2}+4$. For example, as the blue line showed in Step 1 , the $*$ at position $(1,1)$ of $\mathbf{P}$ is put at position $(1,1+2)=(1,3)$ of $\mathbf{C}^{(1)}$, and the $*$ at position $(1,2)$ of $\mathbf{P}$ is put at position $(1,2+4)=(1,6)$ of $\mathbf{C}^{(1)}$. By this construction, any $L$ neighbouring cache-nodes do not cache any common packets.

- Step 2. In the second step, we construct the user-retrieve array $\mathbf{U}^{(1)}$ from $\mathbf{C}^{(1)}$. More precisely, since each user can access $L$ neighbouring cache-nodes in a cyclic wrap-around fashion, we put the stars in $\mathbf{U}^{(1)}$ according to position of the stars in $\mathbf{C}^{(1)}$. In other words, if the entry at the position $(j, k)$ of $\mathbf{C}^{(1)}$ is star, then the entry at the position $\left(j, k_{1}\right)$ of $\mathbf{U}^{(1)}$ is set to be star where $0 \leq k-k_{1} \leq L-1=2$.

- Step 3. In the third step, we construct the user-delivery array $\mathbf{Q}^{(1)}$ from $\mathbf{U}^{(1)}$. First, we let $\mathbf{Q}^{(1)}=\mathbf{U}^{(1)}$. Recall that the null entries in the $k^{\text {th }}$ column represent the required packets in $W_{d_{U_{k}}}^{(1)}$ which can not be retrieved by user $k$ from its connected cache-nodes. For example, the entry at the position $(1,7)$ is null, because user $U_{7}$ requires the first packet of $W_{d_{U_{7}}}^{(1)}$ which can not be retrieved from its connected cache-nodes $C_{7}, C_{8}$ and $C_{1}$. We then fill the null entries in $\mathbf{Q}^{(1)}$ by the integers in $\mathbf{P}$ following the delivery strategy of the MN PDA. Let us focus on the row $j \in[6]$ of $\mathbf{P}$ and $\mathbf{Q}^{(1)}$. Notice that row $j$ of $\mathbf{P}$ contains two integers, and that row $j$ of $\mathbf{Q}^{(1)}$ also contains two nulls. Thus we set the first null to be the first integer and the second null to be the second integer.

After determining $\mathbf{Q}^{(1)}$, it can be seen that $\mathbf{Q}^{(1)}$ satisfies the condition $\mathbf{C} 3$ of Definition 1 . Hence, we use the delivery strategy in Line 9 of Algorithm 1. For example, assume that the request vector is $\mathbf{d}=(1,2, \ldots, 8)$. In the first transmission which corresponds to the integer ' 1 ' in $\mathbf{Q}^{(1)}$, the server sends the XOR of the fourth packet of $W_{1}^{(1)}$ (denoted by $W_{1,4}^{(1)}$ ), the second packet of $W_{4}^{(1)}$ (denoted by $W_{4,2}^{(1)}$ ), and the first packet of $W_{7}^{(1)}$ (denoted by $W_{7,1}^{(1)}$ ), i.e., $W_{1,4}^{(1)} \oplus W_{4,2}^{(1)} \oplus W_{7,1}^{(1)}$.

Finally for each $g \in[8]$, the arrays $\mathbf{C}^{(g)}$ and $\mathbf{Q}^{(g)}$ can be obtained by cyclically right-shifting $\mathbf{C}^{(1)}$ and $\mathbf{Q}^{(1)}$ by $g-1$ positions, respectively. After obtaining $\mathbf{C}^{(g)}$ and $\mathbf{Q}^{(g)}$, the placement and delivery phases in the $g^{\text {th }}$ round can be done as above.

Let $\mathbf{C}=\left[\mathbf{C}^{(1)} ; \mathbf{C}^{(2)} ; \cdots ; \mathbf{C}^{(8)}\right]$ be the total placement array of the cache-nodes. By the construction, each column of $\mathbf{C}$ is a concatenation of all the columns of $\mathbf{C}^{(1)}$; thus each column of $\mathbf{C}$ has exactly 12 stars. In addition, the array $\mathbf{C}$ contains $6 \times 8=48$ rows. Hence, the needed memory size is $M=\frac{12 N}{48}=2$, satisfying the memory size constraint. The total subpacketization of the proposed scheme is 48 , which is equal to the number of rows in $\mathbf{C}$.

Besides the MN PDA, we can also apply the proposed transformation approach to the $\left(K^{\prime}, F^{\prime}, Z, S\right)=(4,2,1,2)$ PDA in [7. Theorem $4(m=1$ and $q=2)]$ or [10, Theorem $3(m=1$ and $q=2, z=1)]$ to obtain a multiaccess coded caching scheme with the subpacketization $F^{\prime} \times K=16$ and load $\frac{S}{F^{\prime}}=1$; to the $\left(K^{\prime}, F^{\prime}, Z, S\right)=(4,4,2,4)$ PDA in [12, Theorem 18 ( $m=2$ and $q=2, t=1)$ ],to obtain a multiaccess coded caching scheme with the subpacketization $F^{\prime} \times K=32$ and load $\frac{S}{F^{\prime}}=1$. 


\section{Proofs of Theorem 1 And Theorem 3}

Let us consider the $(K, L, M, N)$ multiaccess coded caching problem, where $t=\frac{K M}{N} \in\left\{0,1, \ldots,\left\lfloor\frac{K}{L}\right\rfloor\right\}$. In other words, the whole library is totally cached $t$ times in the system. Define that $K^{\prime}=K-t(L-1){ }^{\frac{1}{3}}$ For this multiaccess caching problem, we search a $\left(K^{\prime}, F^{\prime}, Z, S\right)$ PDA $\mathbf{P}=\left(p_{j, k}\right)_{j \in\left[F^{\prime}\right], k \in\left[K^{\prime}\right]}$ for the shared-link caching system where the whole library is also totally cached $t$ times, i.e., $\frac{K^{\prime} Z}{F^{\prime}}=\frac{K M}{N}=t$. We define

$$
\mathcal{A}_{j}=\left\{k: p_{j, k}=*, k \in\left[K^{\prime}\right]\right\}, \forall j \in\left[F^{\prime}\right]
$$

as the column label set of $\mathbf{P}$ where the entries in $j^{\text {th }}$ row are stars. We have $\left|\mathcal{A}_{j}\right|=t$ because each packet is cached exactly $t$ times. So PDA $\mathbf{P}$ should satisfy

- C4. Each row of $\mathbf{P}$ has exactly $t=\frac{K^{\prime} Z}{F^{\prime}}$ stars.

For the $(K, L, M, N)$ multiaccess coded caching problem, we divide each file $W_{n}$ where $n \in[N]$ into $K$ parts with equal length, $W_{n}=\left(W_{n}^{(1)}, \ldots, W_{n}^{(K)}\right)$. Denote the set of the $g^{t h}$ parts by $\mathcal{W}^{(g)}=\left\{W_{1}^{(g)}, \ldots, W_{N}^{(g)}\right\}$, for each $g \in[K]$. As shown in the sketch of the proof in Section III-B, we divide the whole caching procedure into $K$ separate rounds, where in the $g^{t h}$ round we only deal with $\mathcal{W}^{(g)}$. In the $g^{\text {th }}$ round where $g \in[K]$, our construction contains three steps: the generations for the node-placement array $\mathbf{C}^{(g)}$, the user-retrieve array $\mathbf{U}^{(g)}$, and the user-delivery array $\mathbf{Q}^{(g)}$, where the definitions of these three arrays are given in Definition 2 .

\section{A. Placement strategy for cache-nodes: Generation of $\mathbf{C}^{(g)}$ for $g \in[K]$}

The main objective in this step is that each $L$ neighbouring cache-nodes do not cache any common packet. Let us first consider the case where $g=1$. The detailed placement is as follows, which is based on a $\left(K^{\prime}, F^{\prime}, Z, S\right)$ PDA $\mathbf{P}=\left(p_{j, k}\right)_{j \in\left[F^{\prime}\right], k \in\left[K^{\prime}\right]}$ for the shared-link caching system with $K^{\prime}=K-t(L-1)$ and $\frac{K^{\prime} Z}{F^{\prime}}=\frac{K M}{N}$.

We divide each part $W_{n}^{(1)}$ where $n \in[N]$ into $F^{\prime}$ equal-length packets, $W_{n}^{(1)}=\left(W_{n, 1}^{(1)}, \ldots, W_{n, F^{\prime}}^{(1)}\right)$. Then each cache-node $C_{k}$ where $k \in[K]$ caches the following set of packets (recall that $\operatorname{Mod}(b, a) \in\{1, \ldots, a\}$ ),

$$
\begin{aligned}
& \mathcal{Z}_{C_{k}}^{(1)}=\left\{W_{n, j}^{(1)}: k \in \mathcal{C}_{j}^{(1)}, j \in\left[F^{\prime}\right], n \in[N]\right\}, \\
& \text { where } \mathcal{C}_{j}^{(1)}=\left\{\mathcal{A}_{j}[h]+h(L-1): h \in\left[\left|\mathcal{A}_{j}\right|\right]\right\} .
\end{aligned}
$$

Notice that $\mathcal{C}_{j}^{(1)}$ represents the set of cache-nodes which cache the $j^{\text {th }}$ packet of each part in $\mathcal{W}^{(1)}$. As the meaning of stars in PDA, we use the following $F^{\prime} \times K$ node-placement array $\mathbf{C}^{(1)}=\left(c_{j, k}^{(1)}\right)_{j \in\left[F^{\prime}\right], k \in[K]}$, where

$$
c_{j, k}^{(1)}=\left\{\begin{array}{cc}
* & \text { if } k \in \mathcal{C}_{j}^{(1)} \\
n u l l & \text { otherwise }
\end{array},\right.
$$

and each entry at the position $(j, k)$ of $\mathbf{C}^{(1)}$ is star if and only if the packets $W_{n, j}^{(1)}$ for all $n \in[N]$ are cached by cache-node $C_{k}$. By the above construction, for any two distinct integers $h, h^{\prime} \in[t]$ and for any $j \in\left[F^{\prime}\right], g \in[K]$, the following inequality

$$
\left|\mathcal{C}_{j}^{(1)}[h]-\mathcal{C}_{j}^{(1)}\left[h^{\prime}\right]\right|=\left|\mathcal{A}_{j}[h]-\mathcal{A}_{j}\left[h^{\prime}\right]+\left(h-h^{\prime}\right)(L-1)\right| \geq L
$$

holds. Thus any $L$ neighbouring cache-nodes do not cache any common packet.

After determining $\mathbf{C}^{(1)}$, we can obtain $\mathbf{C}^{(g)}$ where $g \in[K]$ by simply cyclically right-shifting $\mathbf{C}^{(1)}$ by $g-1$ positions. Then we let

$$
\mathbf{C}=\left[\mathbf{C}^{(1)} ; \mathbf{C}^{(2)} ; \cdots ; \mathbf{C}^{(K)}\right]
$$

to represent the cached contents of the cache-nodes. Each column of $\mathbf{C}$ is the concatenation of all the columns of $\mathbf{C}^{(1)}$. In addition, by the constraint, the total number of stars in $\left(K^{\prime}, F^{\prime}, Z, S\right)$ PDA equals the total number of stars in $\mathbf{C}^{(1)}$. Hence, the number of stars in each column of $\mathbf{C}$ is $Z K^{\prime}$. The total number of packets of each file equals the number of rows of $\mathbf{C}$, i.e., $K F^{\prime}$. So the needed memory size of each cache-node is

$$
\frac{Z K^{\prime}}{K F^{\prime}} N=\frac{Z}{F^{\prime}} \cdot \frac{K^{\prime}}{K} N=M
$$

satisfying the memory size constraint.

Example 2. Let us return to the example in Section III-B with $K=N=8$ and $L=3$, where the caching procedure is divided into 8 rounds.

\footnotetext{
${ }^{3}$ From Lemmas 4 and 5 when $K \leq t L+1$ the scheme with minimum load is obtained. So we only need to consider the case $K>t L+1$. Then the assumption $K^{\prime}>0$ always holds when $K>t L+1$.
} 
In the first round, we consider $\mathcal{W}^{(1)}=\left\{W_{1}^{(1)}, \ldots, W_{8}^{(1)}\right\}$. Each part $W_{n}^{(1)}$ where $n \in[8]$ is divided into 6 packets, i.e., $W_{n}^{(1)}=\left(W_{n, 1}^{(1)}, W_{n, 2}^{(1)}, \ldots, W_{n, 6}^{(1)}\right)$. By $[15)$ we have

$$
\mathcal{A}_{1}=\{1,2\}, \quad \mathcal{A}_{2}=\{1,3\}, \mathcal{A}_{3}=\{1,4\}, \mathcal{A}_{4}=\{2,3\}, \mathcal{A}_{5}=\{2,4\}, \mathcal{A}_{6}=\{3,4\} .
$$

By 177 we have

$$
\mathcal{C}_{1}^{(1)}=\{3,6\}, \quad \mathcal{C}_{2}^{(1)}=\{3,7\}, \quad \mathcal{C}_{3}^{(1)}=\{3,8\}, \quad \mathcal{C}_{4}^{(1)}=\{4,7\}, \quad \mathcal{C}_{5}^{(1)}=\{4,8\}, \quad \mathcal{C}_{6}^{(1)}=\{5,8\} .
$$

Then each cache-node $C_{k}, k \in[8]$, caches the packets from $\mathcal{W}^{(1)}$ as follows by [16].

$$
\begin{aligned}
\mathcal{Z}_{C_{1}}^{(1)} & =\mathcal{Z}_{C_{2}}^{(1)}=\emptyset ; \\
\mathcal{Z}_{C_{3}}^{(1)} & =\left\{W_{n, 1}^{(1)}, W_{n, 2}^{(1)}, W_{n, 3}^{(1)}: n \in[8]\right\} ; \\
\mathcal{Z}_{C_{4}}^{(1)} & =\left\{W_{n, 4}^{(1)}, W_{n, 5}^{(1)}: n \in[8]\right\} ; \\
\mathcal{Z}_{C_{5}}^{(1)} & =\left\{W_{n, 6}^{(1)}: n \in[8]\right\} ; \\
\mathcal{Z}_{C_{6}}^{(1)} & =\left\{W_{n, 1}^{(1)}: n \in[8]\right\} ; \\
\mathcal{Z}_{C_{7}}^{(1)} & =\left\{W_{n, 2}^{(1)}, W_{n, 4}^{(1)}: n \in[8]\right\} ; \\
\mathcal{Z}_{C_{8}}^{(1)} & =\left\{W_{n, 3}^{(1)}, W_{n, 5}^{(1)}, W_{n, 6}^{(1)}: n \in[8]\right\} .
\end{aligned}
$$

\begin{tabular}{|c|c|c|c|c|c|c|c|}
\hline & \multicolumn{7}{|c|}{ Node-placement arrav $\mathbf{C}^{(2)}$ for $\mathcal{W}^{(2)}$} \\
\hline$n \in[8]$ & $C_{1}$ & $C_{2} \quad C_{3}$ & $C_{4}$ & $C_{5}$ & $C_{6}$ & $C_{7}$ & $C_{8}$ \\
\hline$W_{n, 1}^{(2)}$ & & & * & & & * & \\
\hline$W_{n, 2}^{(2)}$ & & & * & & & & * \\
\hline$W_{n, 3}^{(2)}$ & * & & * & & & & \\
\hline$W_{n, 4}^{(2)}$ & & & & * & & & * \\
\hline$W_{n, 5}^{(2)}$ & * & & & * & & & \\
\hline$W_{n, 6}^{(2)}$ & * & & & & * & & \\
\hline
\end{tabular}

By (18) the above packets cached by cache-nodes can be represented by the first array $\mathbf{C}^{(1)}$ in Table II. which is exactly the array $\mathbf{C}^{(1)}$ in Fig. 4 . To get the array $\mathbf{C}^{(2)}$, we right-shift $\mathbf{C}^{(1)}$ by one position. The total number of stars in $\mathbf{C}^{(1)}$ is 12 , which

TABLE II: Node-placement arrays $\mathbf{C}^{(1)}$ and $\mathbf{C}^{(2)}$.

\begin{tabular}{|c|cccccccc|} 
& \multicolumn{7}{|c|}{ Node-placement array $\mathbf{C}^{(1)}$} & for $\mathcal{W}^{(1)}$ \\
\hline$n \in[8]$ & $C_{1}$ & $C_{2}$ & $C_{3}$ & $C_{4}$ & $C_{5}$ & $C_{6}$ & $C_{7}$ & $C_{8}$ \\
\hline$W_{n, 1}^{(1)}$ & & & $*$ & & & $*$ & & \\
$W_{n, 2}^{(1)}$ & & & $*$ & & & & $*$ & \\
$W_{n, 3}^{(1)}$ & & & $*$ & & & & & $*$ \\
$W_{n, 4}^{(1)}$ & & & & $*$ & & & $*$ & \\
$W_{n, 5}^{(1)}$ & & & & $*$ & & & & $*$ \\
$W_{n, 6}^{(1)}$ & & & & & $*$ & & & $*$ \\
\hline
\end{tabular}

is the total number of stars in the $(4,6,3,4)$ PDA $\mathbf{P}$ in 5 . Then the number of stars in each column of $\mathbf{C}$ is 12 . Since $\mathbf{C}$ has $6 \times 8=48$ rows, the memory ratio of each cache-node is $\frac{M}{N}=\frac{12}{48}=\frac{1}{4}$.

\section{B. Packets retrievable to users: Generation of $\mathbf{U}^{(g)}$ for $g \in[K]$}

Let us also start with $g=1$. Since each user is connected to $L$ neighbouring cache-nodes in a cyclic wrap-around fashion, and can retrieve the cached contents in those cache-nodes. Hence, each user $U_{k}$ where $k \in[K]$ can retrieve the packet $W_{n, j}^{(1)}$ for all $n \in[N]$ if and only if $k$ is an element of the following set

$$
\begin{aligned}
\mathcal{U}_{j}^{(1)} & =\bigcup_{h \in[t]}\left\{\mathcal{C}_{j}^{(1)}[h]-(L-1), \ldots, \mathcal{C}_{j}^{(1)}[h]\right\} \\
& =\bigcup_{h \in[t]}\left\{\mathcal{A}_{j}[h]+(h-1)(L-1), \ldots, \mathcal{A}_{j}[h]+h(L-1)\right\} .
\end{aligned}
$$

Notice that $\mathcal{U}_{j}^{(1)}$ is the set of users who can retrieve the $j^{\text {th }}$ packet of each part in $\mathcal{W}^{(1)}$. Then user $U_{k}$ can retrieve the following packets of $\mathcal{W}^{(1)}$,

$$
\mathcal{Z}_{U_{k}}^{(1)}=\left\{W_{n, j}^{(1)}: k \in \mathcal{U}_{j}^{(1)}, n \in[N]\right\} .
$$

From (19), we showed that any neighbouring cache-nodes do not cache any common packet. Recall that each packet is stored by $t$ cache-nodes and each cache-node is connected to $L$ users. Hence, we have $\left|\mathcal{U}_{j}^{(1)}\right|=t L$ for each $j \in\left[F^{\prime}\right]$, which means that each packet is retrievable by $t L$ users.

From [22], we can generate the $F^{\prime} \times K$ user-retrieve array $\mathbf{U}^{(1)}=\left(u_{j, k}^{(1)}\right)_{j \in\left[F^{\prime}\right], k \in[K]}$, where

$$
u_{j, k}^{(1)}=\left\{\begin{array}{cc}
* & \text { if } k \in \mathcal{U}_{j}^{(1)} \\
\text { null } & \text { otherwise }
\end{array} .\right.
$$


In other words, each entry at position $(j, k)$ of $\mathbf{U}^{(1)}$ is star if and only if the packets $W_{n, j}^{(1)}$ for all $n \in[N]$ can be retrieved by user $U_{k}$.

Remark 1. The number of null entries in $j^{\text {th }}$ row of $\mathbf{U}^{(1)}$ equals the number of integer entries in the $j^{\text {th }}$ row of PDA P since the number of null entries in each $j^{t h}$ row is $K-t L=K-t(L-1)-t=K^{\prime}-t$.

After determining $\mathbf{U}^{(1)}$, we can obtain $\mathbf{U}^{(g)}$ where $g \in[K]$ by simply cyclically right-shifting $\mathbf{U}^{(1)}$ by $g-1$ positions.

Example 3. Let us return to the example in Section III-B. From (21), we have

$$
\begin{array}{lll}
\mathcal{U}_{1}^{(1)}=\{1,2,3,4,5,6\}, & \mathcal{U}_{2}^{(1)}=\{1,2,3,5,6,7\}, & \mathcal{U}_{3}^{(1)}=\{1,2,3,6,7,8\}, \\
\mathcal{U}_{4}^{(1)}=\{2,3,4,5,6,7\}, & \mathcal{U}_{5}^{(1)}=\{2,3,4,6,7,8\}, & \mathcal{U}_{6}^{(1)}=\{3,4,5,6,7,8\} .
\end{array}
$$

From (22), the users can retrieve the packets from $\mathcal{W}^{(1)}$ as follows,

$$
\begin{aligned}
\mathcal{Z}_{U_{1}}^{(1)} & =\left\{W_{n, 1}^{(1)}, W_{n, 2}^{(1)}, W_{n, 3}^{(1)}: n \in[8]\right\} ; \\
\mathcal{Z}_{U_{2}}^{(1)} & =\left\{W_{n, 1}^{(1)}, W_{n, 2}^{(1)}, W_{n, 3}^{(1)}, W_{n, 4}^{(1)}, W_{n, 5}^{(1)}: n \in[8]\right\} ; \\
\mathcal{Z}_{U_{3}}^{(1)} & =\left\{W_{n, 1}^{(1)}, W_{n, 2}^{(1)}, W_{n, 3}^{(1)}, W_{n, 4}^{(1)}, W_{n, 5}^{(1)}, W_{n, 6}^{(1)}: n \in[8]\right\} ; \\
\mathcal{Z}_{U_{4}}^{(1)} & =\left\{W_{n, 1}^{(1)}, W_{n, 4}^{(1)}, W_{n, 5}^{(1)}, W_{n, 6}^{(1)}: n \in[8]\right\} ; \\
\mathcal{Z}_{U_{5}}^{(1)} & =\left\{W_{n, 1}^{(1)}, W_{n, 2}^{(1)}, W_{n, 4}^{(1)}, W_{n, 6}^{(1)}: n \in[8]\right\} ; \\
\mathcal{Z}_{U_{6}}^{(1)} & =\left\{W_{n, 1}^{(1)}, W_{n, 2}^{(1)}, W_{n, 3}^{(1)}, W_{n, 4}^{(1)}, W_{n, 5}^{(1)}, W_{n, 6}^{(1)}: n \in[8]\right\} ; \\
\mathcal{Z}_{U_{7}}^{(1)} & =\left\{W_{n, 2}^{(1)}, W_{n, 3}^{(1)}, W_{n, 4}^{(1)}, W_{n, 5}^{(1)}, W_{n, 6}^{(1)}: n \in[8]\right\} ; \\
\mathcal{Z}_{U_{8}}^{(1)} & =\left\{W_{n, 3}^{(1)}, W_{n, 5}^{(1)}, W_{n, 6}^{(1)}: n \in[8]\right\} .
\end{aligned}
$$

Hence, we can generate the user-retrieve array $\mathbf{U}^{(1)}$ in Table III, which is exactly the array $\mathbf{U}^{(1)}$ in Fig. 4 . To get the array $\mathbf{U}^{(2)}$, we right-shift $\mathbf{U}^{(1)}$ by one position. It can be also seen that the number of null entries in each row of $\mathbf{U}^{(1)}$ equals the number of integer entries in each row of the $(4,6,3,4)$ PDA $\mathbf{P}$.

TABLE III: User-retrieve arrays $\mathbf{U}^{(1)}$ and $\mathbf{U}^{(2)}$.

\begin{tabular}{|c|cccccccc|} 
& \multicolumn{7}{|c|}{ User-retrieve array $\mathbf{U}^{(1)}$} & for $\mathcal{W}^{(1)}$ \\
\hline$n \in[8]$ & $U_{1}$ & $U_{2}$ & $U_{3}$ & $U_{4}$ & $U_{5}$ & $U_{6}$ & $U_{7}$ & $U_{8}$ \\
\hline$W_{n, 1}^{(1)}$ & $*$ & $*$ & $*$ & $*$ & $*$ & $*$ & & \\
$W_{n, 2}^{(1)}$ & $*$ & $*$ & $*$ & & $*$ & $*$ & $*$ & \\
$W_{n, 3}^{(1)}$ & $*$ & $*$ & $*$ & & & $*$ & $*$ & $*$ \\
$W_{n, 4}^{(1)}$ & & $*$ & $*$ & $*$ & $*$ & $*$ & $*$ & \\
$W_{n, 5}^{(1)}$ & & $*$ & $*$ & $*$ & & $*$ & $*$ & $*$ \\
$W_{n, 6}^{(1)}$ & & & $*$ & $*$ & $*$ & $*$ & $*$ & $*$ \\
\hline
\end{tabular}

\begin{tabular}{|c|cccccccc|} 
& \multicolumn{7}{|c|}{ User-retrieve array $\mathbf{U}^{(2)}$ for $\mathcal{W}^{(2)}$} \\
\hline$n \in[8]$ & $U_{1}$ & $U_{2}$ & $U_{3}$ & $U_{4}$ & $U_{5}$ & $U_{6}$ & $U_{7}$ & $U_{8}$ \\
\hline$W_{n, 1}^{(2)}$ & & $*$ & $*$ & $*$ & $*$ & $*$ & $*$ & \\
$W_{n, 2}^{(2)}$ & & $*$ & $*$ & $*$ & & $*$ & $*$ & $*$ \\
$W_{n, 3}^{(2)}$ & $*$ & $*$ & $*$ & $*$ & & & $*$ & $*$ \\
$W_{n, 4}^{(2)}$ & & & $*$ & $*$ & $*$ & $*$ & $*$ & $*$ \\
$W_{n, 5}^{(2)}$ & $*$ & & $*$ & $*$ & $*$ & & $*$ & $*$ \\
$W_{n, 6}^{(2)}$ & $*$ & & & $*$ & $*$ & $*$ & $*$ & $*$ \\
\hline
\end{tabular}

\section{Delivery strategy: Generation of $\mathbf{Q}^{(g)}$ for $g \in[K]$}

We also start with $g=1$. By first letting $\mathbf{Q}^{(1)}=\mathbf{U}^{(1)}$, the next step is to fill the nulls in $\mathbf{Q}^{(1)}$ by some integers. From Remark 1. for each $j \in\left[F^{\prime}\right]$ we can get a new array $\mathbf{Q}^{(1)}$ as follows. We replace the $h^{\text {th }}$ (where $h \in\left[K^{\prime}-t\right]$ ) null entry in the $j^{\text {th }}$ row of $\mathbf{U}^{(1)}$ by the $h^{\text {th }}$ integer entry in the $j^{\text {th }}$ row of $\mathbf{P}$. Specifically, for each $j \in\left[F^{\prime}\right]$, define

$$
\overline{\mathcal{A}}_{j}=\left\{k: p_{j, k} \in[S], k \in\left[K^{\prime}\right]\right\},
$$

i.e., the column label set of $\mathbf{P}$ where the entries in $j^{\text {th }}$ row are integers, and

$$
\overline{\mathcal{U}}_{j}^{(1)}=\left\{k: p_{j, k}=n u l l, k \in[K]\right\},
$$

i.e., the column label set of $\mathbf{U}^{(1)}$ where the entries in $j^{\text {th }}$ row are nulls. From Remark 1 , we have

$$
\left|\overline{\mathcal{A}}_{j}\right|=\left|\overline{\mathcal{U}}_{j}\right|=K^{\prime}-t .
$$

We then define a one-to-one mapping $\psi_{j}$ from $\overline{\mathcal{U}}_{j}^{(1)}$ to $\overline{\mathcal{A}}_{j}$ :

$$
\psi_{j}\left(\overline{\mathcal{U}}_{j}^{(1)}[h]\right)=\overline{\mathcal{A}}_{j}[h], \quad \forall h \in\left[K^{\prime}-t\right], j \in\left[F^{\prime}\right] .
$$


Then the entry of the new array $\mathbf{Q}^{(1)}=\left(q_{j, k}^{(1)}\right)_{j \in\left[F^{\prime}\right], k \in[K]}$ can be written as follows.

$$
q_{j, k}^{(1)}=\left\{\begin{array}{ccc}
s & \text { if } & k \in \overline{\mathcal{U}_{j}^{(1)}} \text { and } p_{j, \psi_{j}(k)}=s ; \\
* & & \text { otherwise. }
\end{array}\right.
$$

Hence, the alphabet set of the resulting $\mathbf{Q}^{(1)}$ consists of $[S]$ and symbol star.

After determining $\mathbf{Q}^{(1)}$, the delivery procedure in the first round is as follows. From Line 9 of Algorithm 1 , for each integer $s \in[S]$ and $g=1$, the server sends the following multicast message

$$
\bigoplus_{q_{j, k}^{(1)}=s, j \in\left[F^{\prime}\right], k \in[K]} W_{d_{U_{k}}, j}^{(1)},
$$

if $\mathbf{Q}^{(1)}$ defined in (26) satisfies the condition C3 in Definition 1, such that each user can directly decode its required packet from each multicast message transmitted by the server.

Now let us discuss the conditions on $\mathbf{P}$ to guarantee that the $\mathbf{Q}^{(1)}$ defined in (26) satisfies the condition $\mathbf{C} 3$ in Definition 1 For any integer $s \in[S]$, assume that there exist two different entries, say, $q_{j_{1}, k_{1}}^{(1)}=q_{j_{2}, k_{2}}^{(1)}=s$. Since the integers in each row of the $\left(K^{\prime}, F^{\prime}, Z, S\right)$ PDA $\mathbf{P}$ are different, then all the integers in each row of $\mathbf{Q}^{(1)}$ must be different by (26). Then we have $j_{1} \neq j_{2}$. From [25) there exist two unique integers $h_{1} \in\left[K^{\prime}-t\right]$ and $h_{2} \in\left[K^{\prime}-t\right]$ satisfying $k_{1}=\overline{\mathcal{U}}_{j_{1}}^{(1)}\left[h_{1}\right]$ and $k_{2}=\overline{\mathcal{U}}_{j_{2}}^{(1)}\left[h_{2}\right]$. So we have $\psi_{j_{1}}\left(k_{1}\right)=\overline{\mathcal{A}}_{j_{1}}\left[h_{1}\right]$ and $\psi_{j_{2}}\left(k_{2}\right)=\overline{\mathcal{A}}_{j_{2}}\left[h_{2}\right]$. Let $k_{1}^{\prime}=\psi_{j_{1}}\left(k_{1}\right)$ and $k_{2}^{\prime}=\psi_{j_{2}}\left(k_{2}\right)$. From the definition of mapping $\psi_{j_{1}}$ and $\psi_{j_{2}}$, we have $p_{j_{1}, k_{1}^{\prime}}=p_{j_{2}, k_{2}^{\prime}}=s$. Let $\left(\mathcal{A}_{j_{1}} \bigcup\left\{k_{1}^{\prime}\right\}\right)\left[i_{1}\right]=k_{1}^{\prime}$ and $\left(\mathcal{A}_{j_{2}} \bigcup\left\{k_{2}^{\prime}\right\}\right)\left[i_{2}\right]=k_{2}^{\prime}$ for some integers $i_{1}$ and $i_{2}$. By (21), 23) and (26), we have $k_{1}=k_{1}^{\prime}+\left(i_{1}-1\right)(L-1)$ and $k_{2}=k_{2}^{\prime}+\left(i_{2}-1\right)(L-1)$. By [23], $q_{j_{1}, k_{2}}^{(1)}=q_{j_{2}, k_{1}}^{(1)}=*$ if and only if $k_{1} \in \mathcal{U}_{j_{2}}^{(1)}$ and $k_{2} \in \mathcal{U}_{j_{1}}^{(1)}$. So $\mathbf{Q}^{(1)}$ defined in 26) satisfies the condition C3 if and only if $\mathbf{P}$ satisfies the following condition.

- C5. For any two distinct entries $p_{j_{1}, k_{1}^{\prime}}$ and $p_{j_{2}, k_{2}^{\prime}}$, if $p_{j_{1}, k_{1}^{\prime}}=p_{j_{2}, k_{2}^{\prime}} \in[S]$, we have

$$
k_{1}^{\prime}+\left(i_{1}-1\right)(L-1) \in \mathcal{U}_{j_{2}}^{(1)} \text { and } k_{2}^{\prime}+\left(i_{2}-1\right)(L-1) \in \mathcal{U}_{j_{1}}^{(1)}
$$

hold where $k_{1}^{\prime}=\left(\mathcal{A}_{j_{1}} \bigcup\left\{k_{1}^{\prime}\right\}\right)\left[i_{1}\right]$ and $k_{2}^{\prime}=\left(\mathcal{A}_{j_{2}} \bigcup\left\{k_{2}^{\prime}\right\}\right)\left[i_{2}\right]$ for some integers $i_{1}, i_{2} \in[t+1]$.

Since the server totally sends $S K$ multicast messages of packets in all rounds and each file is divided into $K F^{\prime}$ packets, the achieved load is $R=\frac{S K}{K F^{\prime}}=\frac{S}{K}$. So the following result can be obtained.

Proposition 1. If a $\left(K^{\prime}, F^{\prime}, Z, S\right)$ PDA $\mathbf{P}$ satisfies $\mathrm{C} 4$ and $\mathrm{C} 5$, there exists a $\left(K=K^{\prime}+t(L-1), L, M, N\right)$ multiaccess coded caching scheme with the memory ratio $\frac{M}{N}=\frac{K^{\prime} Z}{K F^{\prime}}$, subpacketization $F=K F^{\prime}$ and load $R=\frac{S}{F^{\prime}}$.

From Proposition 1, we can use the proposed transformation approach to extend a PDA satisfying C4 and C5 to the multiaccess caching problem. Let us then consider the MN PDA, and we have the following lemma whose proof can be found in Appendix C

Lemma 6. The MN PDA satisfies $\mathrm{C} 4$ and $\mathrm{C} 5$.

Let $\mathbf{P}$ be a $\left(K^{\prime},\left(\begin{array}{c}K^{\prime} \\ t\end{array}\right),\left(\begin{array}{c}K^{\prime}-1 \\ t-1\end{array}\right),\left(\begin{array}{c}K^{\prime} \\ t+1\end{array}\right)\right)$ MN PDA. From Proposition 1, we can get a multiaccess coded caching scheme for the $(K, L, M, N)$ multiaccess caching problem, with the subpacketization $K\left(\begin{array}{c}K^{\prime} \\ t\end{array}\right)=K\left(\begin{array}{c}K-t(L-1) \\ t\end{array}\right)$ and load $R_{1}=\frac{\left(\begin{array}{c}K^{\prime} \\ t+1\end{array}\right) K}{K\left(\begin{array}{c}K^{\prime} \\ t\end{array}\right)}=$ $\frac{K^{\prime}-t}{t+1}=\frac{K-t L}{t+1}$. Hence, we proved Theorem 1

Example 4. Let us return to the example in Section III-B again. From 24] and 25] we have

$$
\overline{\mathcal{A}}_{1}=\{3,4\}, \quad \overline{\mathcal{A}}_{2}=\{2,4\}, \quad \overline{\mathcal{A}}_{3}=\{2,3\}, \quad \overline{\mathcal{A}}_{4}=\{1,4\}, \quad \overline{\mathcal{A}}_{5}=\{1,3\}, \quad \overline{\mathcal{A}}_{6}=\{1,2\},
$$

and

$$
\overline{\mathcal{U}}_{1}^{(1)}=\{7,8\}, \quad \overline{\mathcal{U}}_{2}^{(1)}=\{4,8\}, \quad \overline{\mathcal{U}}_{3}^{(1)}=\{4,5\}, \quad \overline{\mathcal{U}}_{4}^{(1)}=\{1,8\}, \quad \overline{\mathcal{U}}_{5}^{(1)}=\{1,5\}, \quad \overline{\mathcal{U}}_{6}^{(1)}=\{1,2\},
$$

respectively. Then the following mappings can be obtained.

$$
\begin{aligned}
& \psi_{1}(7)=3, \quad \psi_{1}(8)=4, \quad \psi_{2}(4)=2, \quad \psi_{2}(8)=4, \quad \psi_{3}(4)=2, \quad \psi_{3}(5)=3, \\
& \psi_{4}(1)=1, \quad \psi_{4}(8)=4, \quad \psi_{5}(1)=1, \quad \psi_{5}(5)=3, \quad \psi_{6}(1)=1, \quad \psi_{6}(2)=2 .
\end{aligned}
$$

From (26), the user-delivery array $\mathbf{Q}^{(1)}$ can be obtained in Table IV, which is exactly the array $\mathbf{Q}^{(1)}$ in Fig. 4 . To get the array $\mathbf{Q}^{(2)}$, we right-shift $\mathbf{Q}^{(1)}$ by one position. It can be checked that both of $\mathbf{Q}^{(1)}$ and $\mathbf{Q}^{(2)}$ satisfy the condition C3 of Definition 1 Then the load is $R_{1}=\frac{4 \times 8}{6 \times 8}=\frac{2}{3}$.

From Proposition 1, we can use a PDA satisfying C4 and C5 with a low row number to obtain a multiaccess coded caching scheme with a low subpacketization. In Appendix D, we show that the PDA in [7] satisfies C4 and C5. Consequently Theorem 3 can be obtained. Similarly, we can also show that the PDAs in [10], [12], [13] satisfy C4 and C5. 
TABLE IV: User-delivery arrays $\mathbf{Q}^{(1)}$ and $\mathbf{Q}^{(2)}$.

\begin{tabular}{|c|cccccccc|} 
& \multicolumn{1}{|c}{ User-delivery array $\mathbf{Q}^{(1)}$ for $\mathcal{W}^{(1)}$} \\
\hline$n \in[8]$ & 1 & 2 & 3 & 4 & 5 & 6 & 7 & 8 \\
\hline$W_{n, 1}^{(1)}$ & $*$ & $*$ & $*$ & $*$ & $*$ & $*$ & 1 & 2 \\
$W_{n, 2}^{(1)}$ & $*$ & $*$ & $*$ & 1 & $*$ & $*$ & $*$ & 3 \\
$W_{n, 3}^{(1)}$ & $*$ & $*$ & $*$ & 2 & 3 & $*$ & $*$ & $*$ \\
$W_{n, 4}^{(1)}$ & 1 & $*$ & $*$ & $*$ & $*$ & $*$ & $*$ & 4 \\
$W_{n, 5}^{(1)}$ & 2 & $*$ & $*$ & $*$ & 4 & $*$ & $*$ & $*$ \\
$W_{n, 6}^{(1)}$ & 3 & 4 & $*$ & $*$ & $*$ & $*$ & $*$ & $*$ \\
\hline
\end{tabular}

\begin{tabular}{|c|cccccccc|} 
& \multicolumn{1}{|c}{ User-delivery array $\mathbf{Q}^{(2)}$ for $\mathcal{W}^{(2)}$} \\
\hline$n \in[8]$ & 1 & 2 & 3 & 4 & 5 & 6 & 7 & 8 \\
\hline$W_{n, 1}^{(2)}$ & 2 & $*$ & $*$ & $*$ & $*$ & $*$ & $*$ & 1 \\
$W_{n, 2}^{(2)}$ & 3 & $*$ & $*$ & $*$ & 1 & $*$ & $*$ & $*$ \\
$W_{n, 3}^{(2)}$ & $*$ & $*$ & $*$ & $*$ & 2 & 3 & $*$ & $*$ \\
$W_{n, 4}^{(2)}$ & 4 & 1 & $*$ & $*$ & $*$ & $*$ & $*$ & $*$ \\
$W_{n, 5}^{(2)}$ & $*$ & 2 & $*$ & $*$ & $*$ & 4 & $*$ & $*$ \\
$W_{n, 6}^{(2)}$ & $*$ & 3 & 4 & $*$ & $*$ & $*$ & $*$ & $*$ \\
\hline
\end{tabular}

\section{FURTHER IMPROVED TRANSFORMATION APPROACH}

In this section, we will show that based on the placement strategy proposed in Section IV-A we can further reduce the transmission load of the scheme proposed in Section IV-C, if some additional conditions given in the following Proposition 2 are satisfied.

Given a $\left(K^{\prime}, F^{\prime}, Z, S\right)$ PDA, assume $t=\left|\mathcal{A}_{1}\right|=\cdots=\left|\mathcal{A}_{F}^{\prime}\right|$. For each $h \in[t]$, let $a_{h}=\min \left\{\mathcal{A}_{j}[h]: j \in\left[F^{\prime}\right]\right\}$ and $b_{h}=\max \left\{\mathcal{A}_{j}[h]: j \in\left[F^{\prime}\right]\right\}$.

Proposition 2. Given a $\left(K^{\prime}, F^{\prime}, Z, S\right)$ PDA $\mathbf{P}$ satisfying $\mathrm{C} 4$ and $\mathrm{C} 5$, if $\lambda_{h}=a_{h}+L-b_{h}>0$ holds for each $h \in[t]$, then for any positive integers $L, M$ and $N$, there exists a $\left(K=K^{\prime}+t(L-1), L, M, N\right)$ multiaccess coded caching scheme with the memory ratio $\frac{M}{N}=\frac{K^{\prime} Z}{K F^{\prime}}$, subpacketization $F=K F^{\prime}$ and transmission load $R=\frac{S}{F^{\prime}} \cdot \frac{K-\sum_{h=1}^{t} \lambda_{h}}{K}$.

Proof. From Proposition 1, we have a $\left(K=K^{\prime}+t(L-1), L, M, N\right)$ multiaccess coded caching scheme with the memory ratio $\frac{M}{N}=\frac{K^{\prime} Z}{K F^{\prime}}$, subpacketization $F=K F^{\prime}$ and load $R=\frac{S}{F^{\prime}}$. Now let us consider the transmission load by further compressing the multicast messages.

For any integer $j \in\left[F^{\prime}\right]$, we know that

$$
\mathcal{U}_{j}^{(1)}=\bigcup_{h \in[t]}\left\{\mathcal{A}_{j}[h]+(h-1)(L-1), \ldots, \mathcal{A}_{j}[h]+h(L-1)\right\}
$$

is the set of users in $[K]$ who can retrieve the packets $W_{n, j}^{(1)}$ for all $n \in[N]$. So for any two different positive integers $j_{1}$, $j_{2} \in\left[F^{\prime}\right]$, a user $U_{k}, k \in[K]$ can retrieve the packets $W_{n, j_{1}}^{(1)}$ and $W_{n, j_{2}}^{(1)}$ for all $n \in[N]$ if and only if $k \in \mathcal{U}_{j_{1}}^{(1)} \cap \mathcal{U}_{j_{2}}^{(1)}$. For each integer $h \in[t]$,

$$
\begin{aligned}
& \bigcap_{j \in\left[F^{\prime}\right]}\left\{\mathcal{A}_{j}[h]+(h-1)(L-1), \ldots, \mathcal{A}_{j}[h]+h(L-1)\right\} \\
& =\left\{a_{h}+(h-1)(L-1), \ldots, a_{h}+h(L-1)\right\} \bigcap\left\{b_{h}+(h-1)(L-1), \ldots, b_{h}+h(L-1)\right\} \\
& =\left\{b_{h}+(h-1)(L-1), \ldots, a_{h}+h(L-1)\right\},
\end{aligned}
$$

always holds where $[28 \mathrm{~b})$ comes from that $a_{h}=\min \left\{\mathcal{A}_{j}[h]: j \in\left[F^{\prime}\right]\right\}$ and $b_{h}=\max \left\{\mathcal{A}_{j}[h]: j \in\left[F^{\prime}\right]\right\}$. From (28a) and [28c), each user from $\left\{b_{h}+(h-1)(L-1), \ldots, a_{h}+h(L-1)\right\}$ can retrieve all the packets of the first part. From [28c), it can be seen that there are exactly

$$
\sum_{h=1}^{t}\left|\left\{b_{h}+(h-1)(L-1), \ldots, a_{h}+h(L-1)\right\}\right|=\sum_{h=1}^{t} \lambda_{h}
$$

users who can re-construct all the $S$ multicast messages for $\mathbf{Q}^{(1)}$ from their retrieved cache-nodes. By the symmetry, considering all $\mathbf{Q}^{(g)}$ where $g \in[K]$, among all the $K S$ multicast messages in the delivery phase, each user can re-construct $\sum_{h=1}^{t} \lambda_{h} S$ multicast messages. Hence, we can transmit $\left(K-\sum_{h=1}^{t} \lambda_{h}\right) S$ random linear combinations of the $K S$ multicast messages. 4 Then the transmission load is

$$
R=\frac{\left(K-\sum_{h=1}^{t} \lambda_{h}\right) S}{K F^{\prime}}=\frac{S}{F^{\prime}} \cdot \frac{K-\sum_{h=1}^{t} \lambda_{h}}{K}
$$

Then the proof is completed.

Example 5. Let us return to the example in Section III-B with $K=N=8$ and $L=3$, where the caching procedure is divided into 8 rounds. Given the $(4,6,3,4)$ PDA listed in Fig. 4 from (20) we have $a_{1}=1, b_{1}=3, a_{2}=2, b_{2}=4$ and then

\footnotetext{
${ }^{4}$ Instead of random linear combinations, we can also use the parity check matrix of Minnimum Distance Seperable (MDS) code or Cauchy matrix as in [24], to encode the $K S$ multicast messages. In each of these matrices whose dimension is dimension $m_{1} \times m_{2}$ where $m_{1} \leq m_{2}$, every $m_{1}$ columns are linearly independent.
} 
$\lambda_{1}=\lambda_{2}=1>0$. From Proposition 2 there exists a $(K, L, M, N)=(8,3,2,8)$ multiaccess coded caching scheme with the subpacketization $F=K F^{\prime}=48$ and transmission load $R=\frac{S}{F^{\prime}} \cdot \frac{K-\sum_{h=1}^{t} \lambda_{h}}{K}=\frac{4}{6} \cdot \frac{8-2}{8}=\frac{1}{2}$. This transmission load is lower than the transmission load of the scheme in Section III-B (i.e., $\frac{2}{3}$ ).

By (4), in MN PDA we have $\left|\mathcal{A}_{1}\right|=\ldots=\mathcal{A}_{\left(\begin{array}{c}K^{\prime} \\ t\end{array}\right)}=t$ and $a_{h}=h, b_{h}=K^{\prime}-(t-h)$ for each $h \in[t]$. For any positive integer $L$, if

$$
t+1<K^{\prime}<t+L
$$

which implies $t L+1<K<t L+L$ in Theorem 1, we have

$$
\lambda_{h}=a_{h}+L-b_{h}=h+L-\left(K^{\prime}-(t-h)\right)=t+L-K^{\prime}>0 .
$$

From Theorem 1 and Proposition 2 , the following result can be directly obtained.

Corollary 1. For the $(K, L, M, N)$ centralized multiaccess coded caching problem, if $M=\frac{N t}{K}$ where $t \in\left\{0,1, \ldots,\left\lfloor\frac{K}{L}\right\rfloor\right\}$ and $t L+1<K<t L+L$, the following load is achievable,

$$
R_{3}=\frac{K-t L}{t+1} \frac{(t+1)(K-t L)}{K}=\frac{(K-t L)^{2}}{K} .
$$

We can check that the transmission load in Corollary 1 is exactly the transmission load in Lemma 4 This implies that when $t L+1<K<t L+L$, the scheme in [18] has lower transmission load. As shown in (11), when $(t+1) L<K$ the proposed scheme in Theorem 1 has strictly lower transmission load than the scheme in [18].

Now let us consider the scheme in Theorem 3 . We can check that in the PDA from Appendix $|\mathrm{D}| \mathcal{A}_{1} \mid=\ldots=\mathcal{A}_{q^{m-1}}=m$ and $b_{h}-a_{h}=q-1$ for each $h \in[m]$. For any positive integer $L$, if $q \leq L$ in Theorem 3 , we have

$$
\lambda_{h}=L-q+1>0 .
$$

From Theorem 3 and Proposition 2 , we can obtain the following result.

Corollary 2. For any positive integers $m, q \geq 2$ and $L$ with $L \geq q$, there exits an $(m(q+L-1), L, M, N)$ multiaccess coded caching scheme with $\frac{M}{N}=\frac{1}{q+L-1}$, the subpacketization $F=m(q+L-1) q^{m-1}$ and transmission load $R_{4}=\frac{2(q-1)^{2}}{q+L-1}$.

\section{CONCLUSION}

In this paper, we consider the multiaccess coded caching problem and propose a novel transformation approach to extend any shared-link PDA satisfying two conditions to the considered problem. The resulting scheme has the maximum local caching gain and the same coded caching gain as the original PDA. By applying our transformation approach into the MN PDA, the delivery scheme was proved to be approximately optimal when $K$ is sufficiently large, under the constraint of the used placement. Finally we also provided an improved transformation approach to further reduced the load.

On-going works include the generalization of the proposed transformation approach to more general multiaccess topologies, such as the line multiaccess topology where users are connected to different numbers of cache-nodes, the planar multiaccess topology, and the multiaccess coded caching problem with distance-dependent retrieval costs.

\section{APPENDIX A \\ PROOF OF THEOREM 2}

For any integer $q \in[t L: K]$, we define that

$$
\mathcal{S}_{q}=\left\{\mathcal{T} \subseteq[K-q+1: K]:|\mathcal{T}|=t, \operatorname{Mod}\left(j_{1}-j_{2}, K\right) \geq L, j_{1}, j_{2} \in \mathcal{T} \text { and } j_{1} \neq j_{2}\right\} .
$$

It can be computed that

$$
\left|\mathcal{S}_{q}\right|=\left(\begin{array}{c}
q-X \\
t-1
\end{array}\right) \frac{q}{t}
$$

where $X:=t L-t+1$. Thus under the placement in [13) for each $\mathcal{T} \in \mathcal{S}_{K}$ and $i \in[N]$, there is a subfile $W_{i, \mathcal{T}}$ of $W_{i}$ cached by the cache-nodes with indices in $\mathcal{T}$. Each file has $F$ packets and each subfile has $\frac{F t}{\left(\begin{array}{c}K-t L+t-1 \\ t-1\end{array}\right) K}$ packets. 
Consider the demand vector $\mathbf{d}=(1, \ldots, K)$. For each $k \in[K]$, each subfile in $\cup_{\mathcal{T} \in \mathcal{S}_{K-k-L+1}} W_{k, \mathcal{T}}$ is demanded by user $k$ and cannot be retrieved by the users in $[k]$. By the converse bound in [3], [4], we have

$$
\begin{aligned}
& R_{1}^{\star} F \geq \sum_{k \in[K]} \sum_{\mathcal{T} \in \mathcal{S}_{K-k-L+1}\left|W_{q, \mathcal{T}}\right|} \frac{F t}{\left(\begin{array}{c}
K-t L+t-1 \\
t-1
\end{array}\right) K} \sum_{k \in[K]}\left(\begin{array}{c}
K-k-L+1-X \\
t-1
\end{array}\right) \frac{K-k-L+1}{t} \\
& =\frac{F}{\left(\begin{array}{c}
K-t L+t-1 \\
t-1
\end{array}\right) K} \sum_{k \in[K]}(K-k-L+1)\left(\begin{array}{c}
K-k-L+1-X \\
t-1
\end{array}\right) \\
& =\frac{F}{\left(\begin{array}{c}
K-t L+t-1 \\
t-1
\end{array}\right) K}\left(\left(\begin{array}{c}
K-L-X \\
t-1
\end{array}\right)+(K-L-1)\left(\begin{array}{c}
K-L-X+1 \\
t
\end{array}\right)-\left(\begin{array}{c}
K-L-X \\
t+1
\end{array}\right)\right),
\end{aligned}
$$

where (32b) comes from (31) and (32d) comes from the Pascal's triangle. Hence, we prove (14a).

Next, we focus on the case where $K \gg t L$. In this case, we have

$$
\begin{aligned}
& \frac{R_{1}}{R_{1}^{\star}} \leq \frac{(K-t L)\left(\begin{array}{c}
K-t L+t-1 \\
t-1
\end{array}\right) K}{(t+1)\left(\left(\begin{array}{c}
K-L-X \\
t-1
\end{array}\right)+(K-L-1)\left(\begin{array}{c}
K-L-X+1 \\
t
\end{array}\right)-\left(\begin{array}{c}
K-L-X \\
t+1
\end{array}\right)\right)} \\
& \leq \frac{(K-t L)\left(\begin{array}{c}
K-t L+t-1 \\
t-1
\end{array}\right) K}{(t+1)\left((K-L-1)\left(\begin{array}{c}
K-L-X+1 \\
t
\end{array}\right)-\left(\begin{array}{c}
K-L-X \\
t+1
\end{array}\right)\right)} \\
& \leq \frac{(K-t L)\left(\begin{array}{c}
K-t L+t-1 \\
t-1
\end{array}\right) K}{(t+1)\left((K-L-1)\left(\begin{array}{c}
K-L-X+1 \\
t
\end{array}\right)-\frac{K-L-1}{t+1}\left(\begin{array}{c}
K-L-X+1 \\
t
\end{array}\right)\right)} \\
& =\frac{(K-t L)\left(\begin{array}{c}
K-t L+t-1 \\
\text { t }
\end{array}\right)}{t(K-L-1)\left(\begin{array}{l}
K-L-X+1 \\
t
\end{array}\right.} \\
& =\frac{K(K-t L)}{(K-L-1)(K-L-X+1)} \cdot \frac{K-t L+t-1}{K-L-X} \cdot \frac{K-t L+t-2}{K-L-X-1} \cdots \frac{K-t L+1}{K-L-X-t+2} \\
& \leq \frac{K(K-t L)}{(K-L-1)(K-L-X+1)}\left(\frac{K-t L+1}{K-L-X-t+2}\right)^{t-1} \\
& =\frac{K(K-t L)}{(K-L-1)(K-L-t L+t)}\left(1+\frac{L}{K-L-t L+1}\right)^{t-1} \\
& \approx \frac{K(K-t L)}{(K-L-1)(K-L-t L+t)}\left(1+\frac{L(t-1)}{K-L-t L+1}\right) \\
& \approx 1
\end{aligned}
$$

where $33 \mathrm{~h}$ comes from the binomial approximation. Hence, we prove $14 \mathrm{c}$.

\section{APPENDIX B}

PROOF OF (10, , 11) AND (12)

\section{A. Proof of 10,}

We focus on the case where $L$ does not divide $K$. When $M_{1}=\frac{N}{K}\left\lfloor\frac{K}{2 L}\right\rfloor$, we have

$$
\frac{R_{\mathrm{HKD}}}{R_{1}}=\frac{\frac{K-\left\lfloor\frac{K}{2 L}\right\rfloor}{\left\lfloor\frac{K}{2 L}\right\rfloor+1} \cdot \frac{\frac{K}{L}-t}{\frac{K}{L}-\left\lfloor\frac{K}{2 L}\right\rfloor}}{\frac{K-t L}{t+1}}=\frac{K-\left\lfloor\frac{K}{2 L}\right\rfloor}{K-L\left\lfloor\frac{K}{2 L}\right\rfloor} \cdot \frac{t+1}{\left\lfloor\frac{K}{2 L}\right\rfloor+1}>\frac{K-\left\lfloor\frac{K}{2 L}\right\rfloor}{K-L\left\lfloor\frac{K}{2 L}\right\rfloor} .
$$

Let us then focus on a memory size $M_{1} \leq M$. The achieved load by the scheme in Lemma 5 is obtained by memory-sharing between the memory-load tradeoff points $\left(\frac{N}{K}\left\lfloor\frac{K}{2 L}\right\rfloor, R_{\mathrm{HKD}}\right)$ and $\left(\frac{N}{L}, 0\right)$. In addition, the achieved load by the proposed scheme is no worse than the load obtained by memory-sharing between the memory-load tradeoff points $\left(\frac{N}{K}\left\lfloor\frac{K}{2 L}\right\rfloor, R_{1}\right)$ and $\left(\frac{N}{L}, 0\right)$. Additionally with (34), we can prove that with $M$, the multiplicative gap between the achieved loads by Lemma 5 and by the proposed scheme is larger than $\frac{K-\left\lfloor\frac{K}{2 L}\right\rfloor}{K-L\left\lfloor\frac{K}{2 L}\right\rfloor}$, which coincides with 10].

\section{B. Proof of 11}

Let us focus on the case where $K>(t+1) L$. From $(8)$ and $(9)$, we have

$$
\frac{R_{1}}{R_{\mathrm{RK}}}=\frac{K-t L}{t+1} \cdot \frac{K}{(K-t L)^{2}}=\frac{1}{(t+1)\left(1-\frac{t L}{K}\right)}<\frac{1}{(t+1)\left(1-\frac{t}{t+1}\right)}=1 .
$$




\section{Proof of 12}

From Lemma 5 , it can be seen that

$$
R_{\mathrm{SR}} \geq \sum_{h=\frac{K-t L+2}{2}}^{K-t L} \frac{2}{1+\left\lceil\frac{t L}{h}\right\rceil} .
$$

We focus on the non-trivial corner points at the memory sizes $M=\frac{N t}{K}$ where $t \in\left\{1, \ldots,\left\lfloor\frac{K}{L}\right\rfloor\right\}$. We first show that when $\frac{K M}{2 N}\left(1-\frac{M L}{N}\right) \geq 1$, we have $R_{1}<R_{\mathrm{SR}}$. More precisely, we have

$$
\begin{aligned}
& \sum_{h=\frac{K-t L+2}{2}}^{K-t L} \frac{2}{1+\left\lceil\frac{t L}{h}\right\rceil} \geq \sum_{h=\frac{K-t L+2}{2}}^{K-t L} \frac{2}{2+\frac{t L}{h}}
\end{aligned}
$$

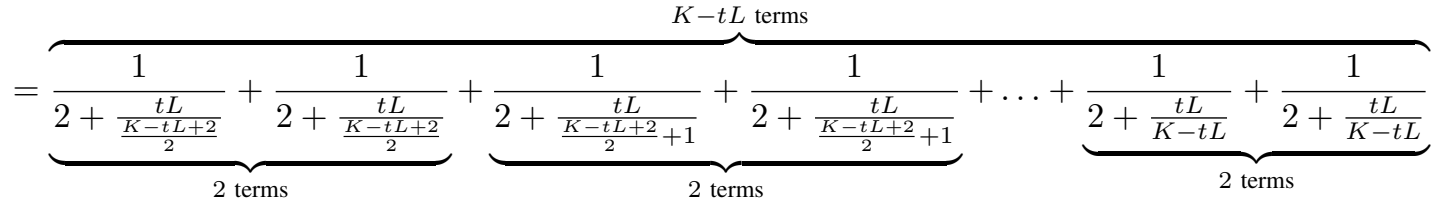

$$
\begin{aligned}
& >(K-t L) \frac{1}{2+\frac{t L}{\frac{K-t L+2}{2}}} .
\end{aligned}
$$

From (35), it can be seen that

$$
\begin{aligned}
& \frac{R_{\mathrm{SR}}}{R_{1}}>\frac{(K-t L) \frac{1}{2+\frac{t L L}{\frac{K-t+2}{2}}}=\frac{t+1}{\frac{K-t L}{t+1}}=\frac{\frac{K M}{N}+1}{2} \cdot \frac{K-\frac{K M L}{N}+2}{K+2}}{=\frac{\frac{K M}{N}+1}{2} \cdot\left(1-\frac{K M L}{N(K+2)}\right)>\frac{\frac{K M}{N}+1}{2} \cdot\left(1-\frac{M L}{N}\right)} \\
& >\frac{K M}{2 N}\left(1-\frac{M L}{N}\right) \geq 1 .
\end{aligned}
$$

Hence, we showed that when $\frac{K M}{2 N}\left(1-\frac{M L}{N}\right) \geq 1$, it holds that $R_{1}<R_{\mathrm{SR}}$.

Let us then consider the regime where $\frac{K M}{2 N}\left(1-\frac{M L}{N}\right)<1$. In this case, we have either $\frac{M}{N}>\frac{1}{2 L}+\sqrt{\frac{1}{4 L^{2}}-\frac{2}{K L}}$ or $\frac{M}{N}<\frac{1}{2 L}-\sqrt{\frac{1}{4 L^{2}}-\frac{2}{K L}}$. When $K \gg L$, we have

$$
\begin{aligned}
& \frac{1}{2 L}+\sqrt{\frac{1}{4 L^{2}}-\frac{2}{K L}} \rightarrow \frac{1}{L} \\
& \text { and } \frac{1}{2 L}-\sqrt{\frac{1}{4 L^{2}}-\frac{2}{K L}} \rightarrow 0 .
\end{aligned}
$$

Recall that $M \leq \frac{N}{L}$. Hence, we can prove that when $K \gg L$, the regime $\frac{K M}{2 N}\left(1-\frac{M L}{N}\right)<1$ does not exist.

In conclusion, if $K \gg L$, we can prove that $R_{1}<R_{\mathrm{SR}}$ for $M=\frac{N t}{K}$ where $t \in\left\{1, \ldots,\left\lfloor\frac{K}{L}\right\rfloor\right\}$.

\section{APPENDIX C}

\section{PROOF OF LEMMA 6}

We can easily check that the MN PDA $\mathbf{P}$ satisfies $\mathrm{C} 4$ by the construction of the MN PDA in (4). We will then prove that C5 holds. For any integer $s \in[S]$, assume that there exist two different integers $j_{1}, j_{2} \in\left[F^{\prime}\right]$ and two different integers $k_{1}^{\prime}$, $k_{2}^{\prime} \in\left[K^{\prime}\right]$, satisfying $p_{j_{1}, k_{1}^{\prime}}=p_{j_{2}, k_{2}^{\prime}}=s$. From the construction of MN PDA in (4) we have $\mathcal{S}=\mathcal{A}_{j_{1}} \cup\left\{k_{1}^{\prime}\right\}=\mathcal{A}_{j_{2}} \cup\left\{k_{2}^{\prime}\right\}$ and $k_{1}^{\prime} \notin \mathcal{A}_{j_{1}}, k_{2}^{\prime} \notin \mathcal{A}_{j_{2}}$. This implies that $k_{1}^{\prime} \in \mathcal{A}_{j_{2}}$ and $k_{2}^{\prime} \in \mathcal{A}_{j_{1}}$. Let

$$
\mathcal{A}_{j_{2}}\left[h_{2}^{\prime}\right]=k_{1}^{\prime}, \quad \mathcal{A}_{j_{1}}\left[h_{1}^{\prime}\right]=k_{2}^{\prime}, \quad k_{1}^{\prime}=\mathcal{S}\left[i_{1}\right], \quad k_{2}^{\prime}=\mathcal{S}\left[i_{2}\right],
$$

for some positive integers $h_{1}^{\prime}, h_{2}^{\prime} \in[t]$ and $i_{1}, i_{2} \in[t+1]$. When $k_{2}^{\prime}<k_{1}^{\prime}$, we have $i_{2}=h_{1}^{\prime}$ and $i_{1}=h_{2}^{\prime}+1$. Then we have

$$
\begin{aligned}
& k_{1}^{\prime}+\left(i_{1}-1\right)(L-1)=\mathcal{A}_{j_{2}}\left[h_{2}^{\prime}\right]+h_{2}^{\prime}(L-1) \\
\in & \left\{\mathcal{A}_{j_{2}}\left[h_{2}^{\prime}\right]+\left(h_{2}^{\prime}-1\right)(L-1), \ldots, \mathcal{A}_{j_{2}}\left[h_{2}^{\prime}\right]+h_{2}^{\prime}(L-1)\right\} \\
\subseteq & \mathcal{U}_{j_{2}}^{(1)}
\end{aligned}
$$




$$
\begin{aligned}
& k_{2}^{\prime}+\left(i_{2}-1\right)(L-1)=\mathcal{A}_{j_{1}}\left[h_{1}^{\prime}\right]+\left(h_{1}^{\prime}-1\right)(L-1) \\
\in & \left\{\mathcal{A}_{j_{1}}\left[h_{1}^{\prime}\right]+\left(h_{1}^{\prime}-1\right)(L-1), \ldots, \mathcal{A}_{j_{1}}\left[h_{1}^{\prime}\right]+h_{1}^{\prime}(L-1)\right\} \\
\subseteq & \mathcal{U}_{j_{1}}^{(1)} .
\end{aligned}
$$

So C5 holds. Similarly when $k_{2}^{\prime}>k_{1}^{\prime}$, we can also show $k_{1}^{\prime}+\left(i_{1}-1\right)(L-1) \in \mathcal{U}_{j_{2}}^{(1)}$ and $k_{2}^{\prime}+\left(i_{2}-1\right)(L-1) \in \mathcal{U}_{j_{1}}^{(1)}$. Then the proof is completed.

\section{APPENDIX D \\ PROOF OF THEOREM 3}

By setting $t=1$ in [25, Construction 1], we have the following shared-link PDA construction.

Construction 1. ( [25, Construction 1]) For any positive integers $m$ and $q \geq 2$, let

$$
\begin{aligned}
& \mathcal{F}^{\prime}=\left\{\left(f_{1}, f_{2}, \ldots, f_{m-1}, f_{m}\right): f_{1}, f_{2}, \ldots, f_{m-1} \in[q], f_{m}=\operatorname{Mod}\left(\sum_{i=1}^{m-1} f_{i}, q\right)\right\}, \\
& \mathcal{K}^{\prime}=\{(\delta, b): \delta \in[m], b \in[q]\} .
\end{aligned}
$$

Then an $\left|\mathcal{F}^{\prime}\right| \times\left|\mathcal{K}^{\prime}\right|$ array $\mathbf{P}=\left(p_{\mathbf{f},(\delta, b)}\right), \mathbf{f} \in \mathcal{F}^{\prime},(\delta, b) \in \mathcal{K}^{\prime}$, can be defined in the following way

$$
p_{\mathbf{f},(\delta, b)}= \begin{cases}\left(\mathbf{e}, n_{\mathbf{e}}\right) & \text { if } f_{\delta} \neq b \\ * & \text { otherwise }\end{cases}
$$

where $\mathbf{e}=\left(e_{1}, e_{2}, \ldots, e_{m}\right) \in[q]^{m}$ such that

$$
e_{i}= \begin{cases}b & \text { if } i=\delta \\ f_{i} & \text { otherwise }\end{cases}
$$

and $n_{\mathbf{e}}$ is the occurrence order of vector e occurs in column $(\delta, b)$.

The following PDA proposed in [25, Theorem 6] can be directly obtained from the above construction.

Lemma 7. ( [25. Theorem 6]) The array $\mathbf{P}$ generated by Construction 1 is an $\left(m q, q^{m-1}, q^{m-2},(q-1) q^{m-1}\right)$ PDA. Furthermore,

- Each row has exactly $m$ stars;

- For each row $\mathbf{f} \in \mathcal{F}^{\prime}$ and each $\delta \in[m]$, there is exactly one star and $q-1$ vector entries in the columns $(\delta, b)$ for all $b \in[q]$.

It is worth noting that the above $\mathbf{P}$ was first constructed in [7, Construction A]. However the rule of defining integer entries in Construction 1 in [25] is much simpler than that of Construction A in [7]. In order to prove our claim easier, we use the notions in [25].

From the first property in Lemma 7, $\mathbf{P}$ satisfies $\mathrm{C} 4$ in Proposition 11 Since each vector $(\delta, b), \delta \in[m]$ and $b \in[q]$, can be uniquely represented by an integer $k=(\delta-1) q+b$. So for convenience of our proof, we will not distinguish to vector $(\delta, b)$ and the integer $(\delta-1) q+b$ in the following. According to [37), the column label set of $\mathbf{P}$, where the entries in $\mathbf{f}^{\text {th }}$ row, $\mathbf{f} \in \mathcal{F}$, are stars, defined in 15 can be written as

$$
\mathcal{A}_{\mathbf{f}}=\left\{(\delta-1) q+f_{\delta}: \delta \in[m]\right\} .
$$

Then the set $\mathcal{U}_{\mathrm{f}}^{(1)}$ defined in 21 can be written as follows.

$$
\mathcal{U}_{\mathbf{f}}^{(1)}=\left\{(\delta-1) L+(\delta-1)(q-1)+f_{\delta}, \ldots, \delta L+(\delta-1)(q-1)+f_{\delta}-1: \delta \in[m]\right\} .
$$

Now let us consider C5 in Proposition 1. For any two different vectors $\mathbf{f}, \mathbf{f}^{\prime} \in \mathcal{F}^{\prime}$ and two different integers $k_{1}^{\prime}, k_{2}^{\prime} \in \mathcal{K}^{\prime}$, assume that $p_{\mathbf{f}, k_{1}^{\prime}}=p_{\mathbf{f}^{\prime}, k_{2}^{\prime}}$ are not star entries. Let

$$
k_{1}^{\prime}=\left(\delta_{1}-1\right) q+b_{1}, \quad k_{2}^{\prime}=\left(\delta_{2}-1\right) q+b_{2}, \quad k_{1}^{\prime}=\left(\mathcal{A}_{\mathbf{f}} \cup\left\{k_{1}^{\prime}\right\}\right)\left[i_{1}\right], \quad k_{2}^{\prime}=\left(\mathcal{A}_{\mathbf{f}^{\prime}} \cup\left\{k_{2}^{\prime}\right\}\right)\left[i_{2}\right],
$$

for some integers $\delta_{1}, \delta_{2} \in[m]$ and $b_{1}, b_{2} \in[q]$ and $i_{1}, i_{2} \in[m+1]$. From the second property in Lemma 7 , until $k_{1}^{\prime}$ we have

$$
\begin{cases}i_{1}=\delta_{1}+1 & \text { if } b_{1}>f_{\delta_{1}} \\ i_{1}=\delta_{1} & \text { if } b_{1}<f_{\delta_{1}}\end{cases}
$$


star entries and then

$$
k_{1}^{\prime}+\left(i_{1}-1\right)(L-1)=\left\{\begin{array}{cc}
\delta_{1} L+\left(\delta_{1}-1\right)(q-1)+b_{1}-1 & \text { if } b_{1}>f_{\delta_{1}} \\
\left(\delta_{1}-1\right) L+\left(\delta_{1}-1\right)(q-1)+b_{1} & \text { if } b_{1}<f_{\delta_{1}}
\end{array} .\right.
$$

Similarly we can get

$$
k_{2}^{\prime}+\left(i_{2}-1\right)(L-1)=\left\{\begin{array}{ll}
\delta_{2} L+\left(\delta_{2}-1\right)(q-1)+b_{2}-1 & \text { if } b_{2}>f_{\delta_{2}}^{\prime} \\
\left(\delta_{2}-1\right) L+\left(\delta_{2}-1\right)(q-1)+b_{2} & \text { if } b_{2}<f_{\delta_{2}}
\end{array} .\right.
$$

Since $\mathbf{P}$ is a PDA, $p_{\mathbf{f}, k_{2}^{\prime}}=p_{\mathbf{f}^{\prime}, k_{1}^{\prime}}=*$ always holds by C3 in Definition 1 Then $b_{1}=f_{\delta_{1}}^{\prime}$ and $b_{2}=f_{\delta_{2}}$ can be derived by (37). By (39) we have

$$
\begin{aligned}
& k_{1}^{\prime}+\left(i_{1}-1\right)(L-1) \\
\in & \left\{\left(\delta_{1}-1\right) L+\left(\delta_{1}-1\right)(q-1)+b, \delta_{1} L+\left(\delta_{1}-1\right)(q-1)+b-1\right\} \\
= & \left\{\left(\delta_{1}-1\right) L+\left(\delta_{1}-1\right)(q-1)+f_{\delta_{1}}^{\prime}, \delta_{1} L+\left(\delta_{1}-1\right)(q-1)+f_{\delta_{1}}^{\prime}-1\right\} \\
\subseteq & \left\{\left(\delta_{1}-1\right) L+\left(\delta_{1}-1\right)(q-1)+f_{\delta_{1}}^{\prime}, \ldots, \delta_{1} L+\left(\delta_{1}-1\right)(q-1)+f_{\delta_{1}}^{\prime}-1\right\} \subseteq \mathcal{U}_{\mathbf{f}^{\prime}}^{(1)} \\
& k_{2}^{\prime}+\left(i_{2}-1\right)(L-1) \\
\in & \left\{\left(\delta_{2}-1\right) L+\left(\delta_{2}-1\right)(q-1)+b_{2}, \delta_{2} L+\left(\delta_{2}-1\right)(q-1)+b_{2}-1\right\} \\
= & \left\{\left(\delta_{2}-1\right) L+\left(\delta_{2}-1\right)(q-1)+f_{\delta_{2}}, \delta_{2} L+\left(\delta_{2}-1\right)(q-1)+f_{\delta_{2}}-1\right\} \\
\subseteq & \left\{\left(\delta_{2}-1\right) L+\left(\delta_{2}-1\right)(q-1)+f_{\delta_{2}}, \ldots, \delta_{2} L+\left(\delta_{2}-1\right)(q-1)+f_{\delta_{2}}-1\right\} \subseteq \mathcal{U}_{\mathbf{f}}^{(1)} .
\end{aligned}
$$

So C5 holds. From Proposition 11, our claim can be obtained. Then the proof is completed.

\section{REFERENCES}

[1] E. Bastug, M. Bennis, and M. Debbah, Living on the edge: The role of proactive caching in 5G wireless networks, IEEE Commun. Magazine, vol. 52, pp. 82-89, Aug. 2014.

[2] M. A. Maddah-Ali and U. Niesen, Fundamental Limits of Caching, IEEE Trans. Inform. Theory, vol. 60, no. 5, pp. 2856-2867, Mar. 2014.

[3] K. Wan, D. Tuninetti, and P. Piantanida, An Index Coding Approach to Caching With Uncoded Cache Placement, IEEE Trans. Inform. Theory, vol. 66, no. 3, pp. 1318-1332, Mar. 2020.

[4] Q. Yu, M. A. Maddah-Ali, and A. S. Avestimehr, The Exact Rate-Memory Tradeoff for Caching With Uncoded Prefetching, IEEE Trans. Inform. Theory, vol. 64, no. 2, pp. 1281-1296, Feb. 2018.

[5] Q. Yu, M. A. Maddah-Ali, and A. S. Avestimehr, Characterizing the Rate-Memory Tradeoff in Cache Networks Within a Factor of 2, IEEE Trans. Inform. Theory, vol. 65, no. 1, pp. 647-663, Jan. 2019.

[6] K. Shanmugam, M. Ji, A. M. Tulino, J. Llorca, and A. G. Dimakis, Finite-Length Analysis of Caching-Aided Coded Multicasting, IEEE Trans. Inform. Theory, vol. 62, no. 10, pp. 5524-5537, Oct. 2016.

[7] Q. Yan, M. Cheng, X. Tang, and Q. Chen, On the Placement Delivery Array Design for Centralized Coded Caching Scheme, IEEE Trans. Inform. Theory, vol. 63, no. 9, pp. 5821-5833, Sep. 2017.

[8] M. Cheng, J. Jiang, X. Tang, and Q. Yan, Some Variant of Known Coded Caching Schemes With Good Performance, IEEE Trans. Commun., vol. 68, no.3, pp. 1370-1377, Mar. 2020.

[9] M. Cheng, J. Jiang, Q. Wang, Y. Yao, A Generalized Grouping Scheme in Coded Caching, IEEE Trans. Commun., vol. 67, no. 5, pp. 3422-3430, May 2019.

[10] M. Cheng, J. Jiang, Q. Yan, X.Tang, Constructions of Coded Caching Schemes With Flexible Memory Size, IEEE Trans. Commun., vol. 67, no. 6, pp. 4166-4176, Jun. 2019.

[11] J. Michel and Q. Wang, Placement Delivery Arrays From Combinations of Strong Edge Colorings, IEEE Trans. Commun, vol.68, no.10, pp. 5953-5964, Oct. 2020.

[12] C. Shangguan, Y. Zhang, and G. Ge, Centralized Coded Caching Schemes: A Hypergraph Theoretical Approach, IEEE Trans. Inform. Theory, vol. 64, no. 8, pp. 5755-5766, Aug. 2018.

[13] Q. Yan, X. Tang, Q. Chen, and M. Cheng, Placement Delivery Array Design Through Strong Edge Coloring of Bipartite Graphs, IEEE Commun. Lett., vol. 22, no. 2, pp. 236-239, Feb. 2018.

[14] X. Zhong, M. Cheng, and J. Jiang, Placement Delivery Array Based on Concatenating Construction, IEEE Communi. Letters, vol. 24, no. 6, pp. 1216-1220, Jun. 2020.

[15] D. Liu, B. Chen, C. Yang, A. F. Molisch, Caching at the wireless edge: design aspects, challenges, and future directions, IEEE Commun. Magazine, vol 54, no. 9, pp. 22-28, Sep. 2016.

[16] K. Shanmugam, N. Golrezaei, A. G. Dimakis, A. F. Molisch, and G. Caire, FemtoCaching: Wireless Content Delivery Through Distributed Caching Helpers, IEEE Trans. Inform. Theory, vol. 59, no. 12, pp. 8402-8413, Dec. 2013.

[17] J. Hachem, N. Karamchandani, and S. N. Diggavi, Coded Caching for Multi-level Popularity and Access, IEEE Trans. Inform. Theory, vol. 63, no. 5, pp. 3108-3141, May 2017.

[18] K. S. Reddy, N. Karamchandani, Rate-Memory Trade-off for Multi-Access Coded Caching With Uncoded Placement, IEEE Trans. Commun, vol. 68, no. 6, pp. 3261-3274, Jun. 2020.

[19] S. Sasi, B. S. Rajan, An Improved Multi-access Coded Caching with Uncoded Placement, arXiv:2009.05377. Sep. 2020

[20] B. Serbetci, E. Parrinello and P. Elia, Multi-access coded caching: gains beyond cache-redundancy, in Proc. IEEE Information Theory Workshop (ITW), Visby, Sweden, 2019, pp. 1-5.

[21] A. A. Mahesh, B. S. Rajan, Coded Caching Scheme with Linear Sub-packetization and its Application to Multi-Access Coded Caching, arXiv:2009.10923 Sep. 2020.

[22] K. S. Reddy and N. Karamchandani, Structured index coding problem and multi-access coded caching, arXiv preprint arXiv:2012.04705 Dec. 2020.

[23] E. Ozfatura and D. Gündüz, Mobility-Aware Coded Storage and Delivery, IEEE Trans. Commun, vol. 68, no. 6, pp. 3275-3285, Jun. 2020.

[24] J. H. van Lint, Introduction to Coding Theory, third version, Springer, 1999, Printed in Germany.

[25] M. Cheng, J. Wang, X. Zhong, and Q. Wang, A unified framework for constructing centralized coded caching schemes, arXiv: 3768116 , May 20. 